\title{
Autophagy determines osimertinib resistance through regulation of stem cell-like properties in EGFR-mutant lung cancer
}

$\mathrm{Li} \mathrm{Li}^{1 \dagger}$, Yubo Wang ${ }^{1 \dagger}$, Lin Jiao ${ }^{1 \dagger}$, Caiyu Lin ${ }^{1}$, Conghua $\mathrm{Lu}^{1}$, Kejun Zhang ${ }^{2}$, Chen Hu ${ }^{1}$, Junyi Ye ${ }^{3}$, Dadong Zhang $^{4}$, Mingxia Feng ${ }^{1}$, Yong $\mathrm{He}^{1}{ }^{*}$

\section{Affiliations:}

${ }^{1}$ Department of Respiratory Disease, Daping Hospital, Army Medical University, Chongqing 400042, China

2 Department of Clinical Laboratory, Daping Hospital, Army Medical University, Chongqing 400042, China

${ }^{3}$ Burning Rock Biotech, Guangzhou 510300, China

4 The Research and Development Institute of Precision Medicine, 3D Medicine Inc., Shanghai 201114, China.

†These authors contributed equally to this work.

Running title: Autophagy and osimertinib resistance

* Corresponding author: Yong He, Department of Respiratory Disease, Daping Hospital, T Army Medical University, Chongqing 400042, China. Phone: 86-23-68757791; Fax: 86-23-68757791; E-mail: heyong8998@126.com

Key words: osimertinib, autophagy, drug resistance, stemness, beclin1

Competing interests: The authors disclosure no potential conflicts of interest. 


\section{ABSTRACT}

28 Drug resistance to Osimertinib, a $3^{\text {rd }}$-generation EGFR-TKI is inevitable. Autophagy plays a

29 contradictory role in resistance of $1^{\text {st }}$ and $2^{\text {nd }}$ generation EGFR-TKI, and its significance in osimertinib 30 resistance is much less clear. We therefore investigated whether autophagy determines osimertinib

31 resistance. First, osimertinib induced autophagy to a much greater extent than that of gefitinib, and autophagy inhibition further increased osimertinib efficacy. Next, enhanced autophagy was found in osimertinib resistant cells and autophagy inhibition partially reversed osimertinib resistance. Enhanced stem-cell like properties were found in resistant cells, and siRNA-knock down of SOX2 or ALDH1Alreversed osimertinib resistance. Of note, autophagy inhibition or siRNA-knock down of

36 Beclin-1 decreased expression of SOX2 and ALDH1A1 and stem-cell like properties. Next, autophagy

37 inhibition and osimertinib in combination effectively blocked tumor growth in xenografts, which was associated with decreased autophagy and stem cell-like properties in vivo. Finally, enhanced autophagy was found in lung cancer patients with resistance to osimertinib. In conclusion, the current study delineates a previously unknown function of autophagy in determining osimertinib resistance through

41 promoting stem-cell like properties. 


\section{Introduction}

Non-small-cell lung cancer (NSCLC) treatment has evolved dramatically in the last decade, from the traditional "one-size-fits-all" chemotherapeutic approach to new targeted therapies against oncogenic driver mutations. In NSCLC patients with EGFR-activating mutations, $1^{\text {st }}$-generation epidermal growth factor receptor tyrosine kinase inhibitors (EGFR-TKIs) have become the standard first-line therapy with dramatic therapeutic efficacy (Nguyen \& Neal, 2012; Soria et al, 2012). However, acquired resistance is unavoidable (Pao et al, 2005). Occurrence of a second EGFR mutation p.T790M in exon 20 represents the most frequent mechanism of acquired resistance(Yu et al, 2013). Osimertinib (AZD9291) is a $3^{\text {rd }}$-generation irreversible EGFR-TKI with potent activities against T790M (Skoulidis \& Papadimitrakopoulou, 2017), and has shown significantly higher efficacy in T790M-positive advanced NSCLC patients (Mok et al, 2017). Moreover, osimertinib showed efficacy superior to that of $1^{\text {st }}$ or $2^{\text {nd }}$ generation EGFR-TKIs in the first-line treatment of EGFR mutation-positive advanced NSCLC (Soria et al, 2018). However, it is very disappointing that acquired resistance to such a highly-effective and low-toxicity drug will inevitably occur (Janne et al, 2015). Thus, innovative treatment strategies are urgently needed to fully clarify the mechanisms of acquired resistance to osimertinib.

The mechanisms of osimertinib resistance are diverse and not fully understood. Emerging clinical data suggest that the underlying mechanisms include other EGFR mutations, C797S and L798I, which also prevent drug binding(Chabon et al, 2016; Thress et al, 2015), bypassing of MET or ERBB2 signaling activation(Kim et al, 2015; Mizuuchi et al, 2016; Ortiz-Cuaran et al, 2016; Planchard et al, 2015), or constitutive MAPK pathway activation by mutated KRAS or MEK(Eberlein et al, 2015). Besides, amplification of EGFR wild-type alleles but not mutant alleles is sufficient to confer acquired resistance to osimertinib(Nukaga et al, 2017). However, the majority of patients likely develop 
resistance by as yet unknown mechanisms. Therefore, it is of great significance to investigate new treatment regimens which can reverse osimertinib resistance caused by diverse mechanism and enhance osimertinib efficacy.

Autophagy is an evolutionarily conserved catabolic process involving the degradation of cytoplasmic constituents, and the recycling of long-lived or aggregated proteins(Yu et al, 2017). In multiple tumor cells, autophagy is upregulated during adverse conditions, including chemoradiotherapy or a nutrient-deficient environment, promoting tumor cell survival; thus, autophagy may be considered a potential mechanism of drug resistance(2014; Auberger \& Puissant, 2017; Chen et al, 2016b). In NSCLC treated with EGFR-TKI, autophagy is a double-edged sword contributing to both cell survival and death. Reduced autophagy was related to resistance to erlotinib therapy (Wei et al, 2013). On the other hand, several recent studies have shown that treatment of erlotinib or afatinib induced autophagy and inhibition of autophagy improves the anti-tumor activity of these drugs in lung adenocarcinoma (Hu et al, 2017; Wang et al, 2016). Moreover, the pro-cell survival and pro-cell death roles of autophagy can be switched by adding gefitinib at an early time of hypoxia or by re-activating EGFR at a later time of hypoxia in cancer cell lines (Chen et al, 2016a). Therefore, more works are needed to better understand the role of autophagy in EGFR-targeted therapy for NSCLCs. As a $3^{\text {rd }}$ generation EGFR-TKI, osimertinib has a different chemical structure and different potential resistance mechanisms when compared to $1^{\text {st }}$ or $2^{\text {nd }}$ generation EGFR-TKI. Recently, in osimertinib-sensitive cells, osimertinib was found to induce autophagy (Tang et al, 2017). However, it is unknown whether the accumulated autophagy may induce osimertinib resistance, or whether inhibition of autophagy may restore osimertinib sensitivity.

Therefore, we preformed the current study to clarify the role of autophagy in osimertinib resistance 
86 and the potential mechanisms. Since use of osimertinib in $1^{\text {st }}$ or $2^{\text {nd }}$ line may lead to different resistance 87 mechanisms, a series of cell lines were chosen to mimic the clinical usage of osimertinib, including 88 PC-9 cells (19del and sensitive to $1^{\text {st }}$ generation EGFR-TKIs), PC-9GR cells (T790M+ with acquired 89 resistance to the $1^{\text {st }}$ generation EGFR-TKI gefitinib), and H1975 cells (de novo T790M+ with primary 90 resistance to gefitinib), respectively. We first found that osimertinib treatment increased autophagy to a 91 much greater extent than that of gefitinib in osimertinib-sensitive cells, and autophagy inhibitors act 92 synergistically with osimertinib to inhibit cell growth. Next, we demonstrated that enhanced autophagy 93 was a common feature in osimertinib-resistant cells with heterogeneous mutations. Inhibition of 94 autophagy reversed osimertinib resistance. Mechanistically, beclin 1-mediated autophagy determined 95 osimertinib resistance through regulation of stem-cell like properties by upregulating Sox2 and ALDH1, 96 which indeed promote osimertinib resistance. Clinically, enhanced autophagy was also found in several 97 patients with resistance to osimertinib. These findings highlight the importance of beclin 1-mediated autophagy in acquired resistance to osimertinib. 


\section{Results}

\section{Enhanced autophagy lead to drug resistance in osimertinib-sensitive cells}

We first interrogated whether osimertinib treatment could induce autophagy and the role of autophagy in osimertinib sensitivity. PC-9GR and PC-9 cells were treated with osimertinib, and then exposed to cyto-ID green detection reagent that selectively labels accumulated autophagic vacuoles. More pre-autophagosomes, autophagosomes, and autolysosomes were observed after osimertinib treatment in PC-9GR and PC-9 cells. Increased LC3 II expression and decreased p62 expression were also found in both cell lines after osimertinib treatment (Fig. 1A and B, Fig. S1). To further confirm whether autophagy was induced after osimertinib treatment, we examined the autophagic flux in osimertinib-treated PC-9 cells using MG132, a potent proteasome inhibitor. Result showed that LC3II was further significantly increased in both cell lines under osimertinib plus MG132 combination treatment compared to osimertinib alone, indicating that osimertinib induced high autophagic flux (Fig. 1B, Fig. S2A). Interestingly, the level of osimertinib-induced autophagy was much higher than that of gefitinib in PC-9 and PC-9GR cells (Fig. 1C, Fig. S2B). Considering previous reports that autophagy play a complex role in gefitinib or erlotinib resistance, we next investigated whether the highly elevated autophagy could affect osimertinib sensitivity. We observed that treatment with SP-1, a specific and potent autophagy inhibitor, abolished osimertinib-induced autophagy increasement and significantly increased osimertinib sensitivity in both cell lines, as determined by the MTT assay (Fig. 1D, Fig. S3). Similar results were obtained with two other autophagy inhibitors, 3-MA (3-Methyladenine, a PI3K inhibitor) and CQ (chloroquine diphosphate salt, which inhibits the integration of autophagosomes with lysosomes) (Fig. S4). The Ki67 incorporation assay revealed that SP-1 combined with osimertinib resulted in a robust inhibition of cell proliferation in PC-9GR cells 
(Fig. 1E). These results showed that osimertinib treatment induced autophagy in osimertinib-sensitive cells, and inhibition of autophagy increased osimertinib efficacy. We then investigated whether autophagy enhancement by rapamycin, the prototypic inhibitor of mammalian target of rapamycin (mTOR), could decrease osimertinib sensitivity. MTT assay showed that treatment with rapamycin resulted in decreased osimertinib sensitivity (Fig. 1F), and increased autophagy was confirmed by LC3II and p62 level alterations (Fig. 1G, Fig. S5). Taken together, we conclude that autophagy plays an important role in osimertinib sensitivity.

\section{Generation of osimertinib-resistant cell lines}

In order to investigated whether autophagy play a role in osimertinib resistance, osimertinib-resistant cell lines were established from PC-9GR cells, H1975 cells and PC-9 cells, respectively, using the single-colony selecting method. The origins of the parental cells were confirmed by the STR loci assay. The osimertinib-resistant cells (PC-9OR, PC-9GROR and H1975-OR cells) displayed similar morphologic features compared to parental cells (Fig. 2A, Fig. S6). Next, the MTT assay was performed to evaluate osimertinib resistance of the cell lines. As shown in Fig. 2B, they were all highly-resistant to osimertinib, with $\mathrm{IC}_{50}$ markedly elevated when compared with those of parental cells. Next, the long-term proliferation ability of the resistant cells was evaluated by colony formation assay. Under osimertinib pressure, colonies were found in osimertinib-resistant cells but not in parental cells (Fig. 2C, Fig. S7). These results indicated that these cell lines generated were highly resistant to osimertinib.

To clarify potential resistance mechanisms, DNA isolated from parental and resistant clones were subjected to whole exome sequencing. Several genetic alterations potentially relevant to osimertinib 
resistance were identified (Fig. 2D). EGFR amplification and loss of T790M were identified in PC-9GROR3 cells, while $M E T$ amplification was found in all H1975-OR cells; BRAF amplification was detected in H1975-OR1 cells (Fig. 2D). However, no new mutations were found in PC-9OR cells. These results indicated that diverse mechanisms may exist in osimertinib resistance.

\section{Enhanced autophagy in osimertinib-resistant cell lines determines resistance to osimertinib}

We next estimated autophagy levels in osimertinib-resistant cell lines. Increased numbers of accumulated autophagic vacuoles were found in these cells, as shown by fluorescence levels quantified using high-content imaging system (Fig. 3A, Fig. S8). Meanwhile, the protein levels of LC3II was upregulated and p62 was downregulated in PC-9GROR, PC-9OR and H1975-OR cells (Fig. 3B, Fig. S9). Next, we compared autophagy flux in parental and resistant cells. Under MG132 treatment, LC3 II level was highly increased in PC-9OR3 cells when compared with that of parental PC-9 cells (Fig.3 C, Fig. S10), indicating high autophagy flux in osimertinib-resistant cells. To further confirm autophagy completion, osimertinib-resistant cells and respective parental cells were assessed by transmission electron microscopy. Autolysosomes, which have a single limiting membrane and contain cytoplasmic/organellar materials at various stages of degradation, can be distinguished from autophagosomes (containing a double limiting membrane) by electron microscopy. More autolysosomes were found in PC-9GROR3, PC-9OR3 and H1975-OR3 cells, compared with respective parental cells (Fig. 2D). We further examined the relative levels of autophagic flux using mCherry-EGFP-LC3 in PC-9GR and PC-9GROR cells. After transfection, autophagosomes were shown as yellow punta (the combination of red and green fluorescence), and autolysosomes were shown as red punta (the extinction of EGFP in the acid environment of lysosomes). As shown in Figure E, both the number of yellow 
autophagosomes and red autolysosomes (the extinction of EGFP in the acid environment of lysosomes) were increased in the PC-9GROR cells compared to PC-9GR cells. These observations indicated that the autophagic process was completed, rather than blocked at the fusion step.

Next, we examined the role of enhanced autophagy in osimertinib resistance through pharmacological inhibition of autophagy. SP-1 treatment suppressed autophagy in osimertinib-resistant PC-9GROR, PC-9OR and H1975-OR cells, as shown by decreased LC3 II levels and increased p62 amounts (Fig. S11, 12, 13). Importantly, treatment with SP-1 re-sensitized the resistant cells to osimertinib (Fig. 2E, Fig. S11, 12, 13). Another autophagy inhibitor CQ was also used to treat PC-9OR3 cells, and the cells became more sensitive to osimertinib (Fig. S14). Taken together, autophagy was enhanced in osimertinib-resistant cell lines, and inhibition of autophagy re-sensitized those cells to osimertinib.Osimertinib-resistant cells have typical stem cell-like properties

Cancer stem-like cells contribute to tumor heterogeneity and have been implicated in disease relapse and drug resistance (Yeo et al, 2016). To investigated the potential role of stem cell-like properties in osimertinib resistance, pulmosphere formation assay were performed. As expected, PC-9GROR, PC-9OR and H1975-OR cells displayed increased pulmospheres in terms of number and size, comparing to PC-9GR, PC-9 and H1975 cells, respectively (Fig. 4A, Fig. S15). Moreover, CD133 and CD44 are regarded as specific markers for stem-like cells in lung cancer (Nishino et al, 2017; Okudela et al, 2012; Sarvi et al, 2014). FACS analysis revealed that CD133- and CD44-positive cell populations in all osimertinib-resistant cells (PC-9OR, PC-9GROR and H1975-OR) was higher than their parental cells (Fig. 4B). In addition, it was reported that the transcription factor Sox 2 and aldehyde dehydrogenase (ALDH) play major roles in stem-like NSCLC cells (Akunuru et al, 2012; Justilien et al, 2014; Sterlacci et al, 2014), either of which is thought to confer drug resistance to tyrosine kinase inhibitors(Dogan et al, 
2014; Kim et al, 2014). In our study, higher Sox2 and ALDH1 levels were observed in osimertinib resistant cells when compared to their parental cells (Fig. 4C, Fig. S16). Taken together, these results demonstrated that osimertinib-resistant cells exhibited stem-cell like properties such as enhanced pulmosphere formation ability, higher CD133/CD44 enrichment as well as Sox2 and ALDH1 overexpression.

\section{Roles of Sox2 and ALDH1 in the maintenance of CSCs and osimertinib resistance}

To validate the roles of Sox2 and ALDH1 in stemness, small interfering RNAs targeting SOX2 and ALDHIA1 respectively, were designed to investigated the sensitivity of PC-9OR3 cells to osimertinib. First, MTT assay revealed that PC-9OR3 cells were more sensitive to osimertinib after knockdown of either SOX2 or ALDHIA1 (Fig. 5A). Secondly, the pulmosphere formation assay showed that SOX2 or ALDHIA1 knockdown significantly reduced the number and size of pulmospheres compared with controls (Fig. 5B). Thirdly, colony formation assay demonstrated that SOX2 or ALDHIAl knockdown resulted in significantly decreased clone sizes, suggesting that Sox 2 and ALDH1 were responsible for the proliferation of resistant cells (Fig. 5B). Fourthly, PC-9OR3 cells transfected with either SOX2 or ALDHIA1 siRNA displayed decreased CD133+ and CD44+ cell populations (Fig. 5C). These findings indicated that Sox2 and ALDH1 might be essential for maintaining stemness and resistance to osimertinib. In addition, ALDH1 protein levels decreased after silencing of $S O X 2$, whereas no Sox2 protein levels change was observed after ALDHIAl knockdown (Fig. 5D, Fig. S17). These findings demonstrated that the role of ALDH1 in maintaining stemness and osimertinib resistance might be mediated by Sox 2 . 
212 Beclin 1-dependent, not Atg5-related autophagy maintains stem-like cell properties in

\section{osimertinib-resistant cells}

We next investigated the mechanism of autophagy induced osimertinib resistance by maintaining stem cell-like properties. SP-1 treatment lead to smaller spheres PC-9OR3 cells, compared to control (Fig. 6A and Fig. S18). Flow cytometry also revealed that SP-1 resulted in decreased population rates of CD133 and CD44 positive cells (Fig. 6B, Fig. S19). These findings indicated autophagy inhibition can decrease stem cell-like characteristics in osimertinib-resistant cells. In addition, we found that SP-1 treatment downregulated ALDH1 and Sox2 in osimertinib-resistant cells (PC-9OR1, 2, 3) (Fig. 6C, Fig. S20). Similar observations were obtained in PC-9GROR3 cells and H1975-OR3 cells (Fig. S21). These results demonstrated that autophagy inhibition can result in decreased stemness in osimertinib-resistant cells.

Atg5 is an essential gene in canonical macroautophagy, while the non-canonical autophagic pathway, which is independent of Atg5, has been reportede (Honda et al, 2014; Ma et al, 2015). Next, we investigated whether Atg5-dependent autophagy maintains stem cell-like characteristics. Atg5 and phosphorylated beclin 1 (Ser 93) levels were increased in resistant cells, while total Beclin 1 expression remained unchanged (Fig. 6D and Fig.S22). Similar results were found in other osimertinib resistant cells (Fig. S23). Treatment with SP-1 resulted in decreased beclin 1 phosphorylation but not total beclin 1 and Atg5 amounts (Fig. S24). We silenced Atg5 and beclin 1 by siRNAs to examine their effects on osimertinib resistance. Knockdown of beclin 1 resulted in enhanced sensitivity of PC-9OR3 cells to osimertinib, whereas Atg5 knockdown showed no remarkable effects (Fig. 6E, Fig. S25). Furthermore, colony and pulmosphere formation assays demonstrated that beclin 1, not Atg5, was essential for stem cell-like properties, as decreased colony formation and smaller pulmospheres were observed only in beclin 1 knockdown cells (Fig. 6F). In addition, siRNA targeting beclin 1 led to a significant decrease of 
234 CD133/CD44-positive cells while siRNA targeting Atg5 showed no significant effects (Fig. 6G, Fig. 235 S26).

Next, the effects of beclin 1 knockdown on Sox 2 and ALDH1 protein levels were evaluated.

237 Results showed that beclin 1 knockdown resulted in decreased Sox2 and ALDH1 protein amounts (Fig.

6H, Fig. S27), and beclin 1 knockdown also weakened the accumulation of Sox 2 and ALDH1 proteins

after the proteasome inhibitor MG132 treatment (Fig. 6I, Fig. S28). Interestingly, mRNA levels of SOX2

and $A L D H 1 A 1$ were unchanged after beclin 1 knockdown (Fig. 6J). This suggested that beclin1, but not

Atg5, might maintain stemness through preventing the protein degradation of Sox 2 and ALDH in osimertinib-resistant cells.

\section{Autophagy inhibition enhances the anti-tumor activity of osimertinib in PC-9GR/mouse}

xenografts

We next assessed whether combination of the autophagy inhibitor CQ and osimertinib is more

effective in xenografts established with PC-9GR cells. Result showed that CQ treatment slightly reduced tumor growth in PC-9GR xenografts, and osimertinib alone resulted in significant tumor shrinkage. The combination of $\mathrm{CQ}$ and osimertinib can further inhibit tumor growth $(\mathrm{P}<0.05$ compared with osimertinib alone; Fig. 7A). During the treatment, no overt weight loss was observed in mice treated with CQ and/or osimertinib (Fig. 7B). Collectively, these findings suggested that CQ enhanced the therapeutic efficacy of osimertinib in vivo.

Next we explored the mechanism of combined therapy which was more effective than monotherapy in PC-9GR xenografts. Immunohistochemical staining showed high expression of LC3 and low Sox2 levels in the combination group (Fig. 7C). Osimertinib treatment resulted in increased Beclin-1 
256 phosphorylation, while the combination therapy decreased Beclin-1 and Sox2 phosphorylation (Fig. 257 7D). These findings suggested that CQ/osimertinib combination was associated with the inhibition of autophagy and stem cell like properties in vivo.

\section{Enhanced autophagy was found in NSCLC patients with resistance to osimertinib}

Next, we investigated whether enhanced autophagy existed in NSCLC patients with resistance to

osimeritnib. A retrospective analysis was performed by enrolling 39 NSCLC patients who had developed drug resistance to osimertinib from August 2015 to Feb 2018 in our hospital. Prior to treatment of osimertinib, all these patients displayed resistance to 1st-generation EGFR-TKI and EGFR T790M mutation was detected in them. After osimertinib resistance, either plasma or tissue biopsies from these 39 patients were profiled by capture-based targeted ultra-deep sequencing. As shown in Fig. 8A, a series of potential resistance mechanisms were found, including EGFR C797S mutation, MET amplification, ERBB2 amplification, KRAS mutation, PI3K mutation, et al. Of note, 57\% patients developed resistance with unknown mechanisms. We next examined LC3 expression in 5 patients with paired tumor tissue samples (before osimertinib treatment and after osimertinib resistance). Before osimertinib treatment, low LC3 expression was found in all 5 patients (Fig. 8B). After osimertinib resistance, elevated LC3 expression was found in 3 patients (Patient \#1, 4 and 5). Overall mutation spectrum of the 5 patients was displayed in Fig. 8C. Of the 3 patients with increased LC3 expression after osimertinib resistance, EGFR C797S mutation was found in 1 patient, sensitive EGFR mutations were found in the other 2 patients. In the remaining 2 patients without LC3 level increasing, C-met amplification was identified. Taken together, these results indicate that enhanced autophagy exist in at least some NSCLC patients with resistance to osimertinib. 


\section{Discussion}

Currently there is no effective approach to overcome acquired resistance to $3^{\text {rd }}$-generation EGFR-TKI osimertinib. The current study demonstrated that enhanced autophagy not only induced drug resistance in osimertinib-sensitive cells, but also was a general feature in osimertinib-resistant cells which presents diverse and heterogeneous mutations. Autophagy inhibitors and osimertinib synergistically inhibited the growth of both sensitive and resistant tumor cells. Enhanced stem-cell like properties were found in osimertinib-resistant cells. Of note, beclin 1-mediated autophagy helped maintain stem cell-like properties by upregulating Sox2 and ALDH1, which indeed facilitate osimertinib resistance. CQ in combination with osimertinib significantly inhibited tumor growth in xenograft experiments. Taken together, we have shown that pro-survival autophagy determines osimertinib resistance through regulation of stem-cell like properties.

Role of autophagy in lung cancer targeted therapy is perplexing. In advanced lung adenocarcinoma treated with gefitinib, ATG5 rs510532 and ATG10 rs10036653 genetic variations in autophagy core genes are significantly associated with clinical outcomes (Yuan et al, 2017). Previously, reduced autophagy was related to resistance to erlotinib therapy (Wei et al, 2013), and when autophagy is further elevated by a treatment in addition to $2^{\text {nd }}$ generation EGFR-TKI afatinib, it can induce autophagic cell death (Lee et al, 2015). On the other hand, reports demonstrated that gefitinib and erlotinib induced pro-cell survival autophagy in both sensitive and resistant cancer cells (Han et al, 2011; Sugita et al, 2015; Zou et al, 2013). Combining glucose deprivation and autophagy inhibitor could synergize and overcome the acquired resistance against erlotinib (Ye et al, 2017). Taken together, the role of autophagy in resistance to $1^{\text {st }}$ and $2^{\text {nd }}$ generation EGFR-TKI is contradictory. The chemical structure of osimertinib is totally different from $1^{\text {st }}$ and $2^{\text {nd }}$ generation EGFR-TKI, and the role of autophagy in osimertinib 
resistance is unknown.

In the current study, we found a striking difference between gefitinib and osimertinib-induced autophagy. In gefitinib-resistant PC-9GR cells, the level of autophagy was only slightly higher than that of parental PC-9 cells, while a much higher level of autophagy was found in osimertinib-resistant cell lines than their parental cells. Moreover, in both PC-9 cells and PC-9GR cells, osimertinib induced autophagy to a much greater extent than that of gefitinib. Significantly, inhibition of autophagy by several inhibitors and si-RNAs in vitro resulted in enhanced osimertinib efficacy, and the combination of $\mathrm{CQ}$ and osimertinib in vivo markedly decreased tumor growth than osimertinib alone. Clinically, enhanced autophagy was found in several patients with resistance to osimertinib. These results indicate that pro-cell survival autophagy leads to osimertinib resistance. Previously, activation of pro-survival autophagy has been found in therapeutics of many cancer, and blockage of autophagy promotes cell death (Amrein et al, 2011; Han et al, 2008; Lee et al, 2017). Inhibition of autophagy has been proposed as a new approach to enhance efficacy of targeted therapy. For example, simultaneously targeting Hedgehog signaling pathway and autophagy could overcome drug resistance of BCRABL-positive chronic myeloid leukemia to imatinib (Zeng et al, 2015). Elevated autophagy activity contributes to the enhanced tolerance to metabolic stresses of EGFRvIII-expressing cells in glioblastoma. Targeting this survival mechanism abrogates this advantage and results in enhanced tumor cell killing (Jutten et al, 2018). Taken together, our results with those findings suggest that pro-cell survival autophagy plays an important role in targeted therapy of cancer.

Targeting autophagy may be developed as a new approach to overcome osimertinib resistance clinically. The current study established osimertinib-resistant cell lines from PC-9 cells, which have only a sensitive EGFR mutation, and PC-9GR and H1975 cells, in which T790M is present. This choice of 
cells reflected the clinical application of osimertinib in $1^{\text {st }}$ or $2^{\text {nd }}$ line settings. Besides, loss of T790M,

EGFR amplification, Met amplification, and BRAF amplification were found in osimertinib-resistant

cell lines, in line with the clinical situation that diverse mutations of known driver genes and unknown

mechanisms faced by patients with osimertinib resistance (as shown in Fig. 8A). Interestingly, enhanced

autophagy were found in all resistant cell lines and several patients with different potential resistance mechanisms to osimertinib, which indicates that autophagy inhibition may be effective in osimertinib-resistant patients with heterogeneous resistance mechanisms. In the current study, we found that $\mathrm{CQ}$ in combination with osimertinib in vivo markedly decreased tumor growth. CQ is an FDA approved drug used for malaria, rheumatoid arthritis, and other autoimmune diseases, and is very cheap with an established history of good tolerability. Therefore, CQ may be applied together with osimertinib clinically to enhance osimertinib efficacy or to overcome osimertinib resistance.

The underlying mechanism of how autophagy renders osimertinib resistance is unknown. The importance of stemness in tumor heterogeneity and the heterogeneity of resistance mechanisms found in osimertinib-resistant cell lines in the current study initiated us to investigate whether autophagy may regulate osimertinib resistance through regulation of stem cell-like properties. In fact, osimertinib-resistant cells exhibited stem-cell like properties of enhanced pulmosphere formation ability, high CD133/CD44 enrichment as well as Sox2 and ALDH1 overexpression. Moreover, siRNA knockdown of SOX2 or ALDH1A1 increased osimertinib sensitivity, decreased CD133+ and CD44+ populations as well as pulmosphere formation ability. These results indicate that Sox2-mediated ALDH1 expression was involved in maintaining stemness and conferring osimertinib resistance. Previously, 
2013). Also, stem cell-like characteristics were found in gefitinib-resistant cells, and knockdown of IL-8 led to loss of stem cell-like characteristics and enhanced gefitinib sensitivity (Liu et al, 2015). Therefore, our results, together with previous findings, indicate that enhanced stem cell-like properties mediate osimertinib resistance.

We next asked whether autophagy controls osimertinib resistance through regulation of stem cell-like properties. Previously, it was reported that autophagy suppresses hematopoietic stem cell metabolism by clearing active, healthy mitochondria to maintain stemness (Ho et al, 2017). Here, we addressed the importance of Beclin 1 in maintaining stem-cell like properties. We found that beclin 1 knockdown by siRNA resulted in complete suppression of stem-cell like properties (decreased formation of pulmospheres and reduced levels of the stem cell markers Sox2, ALDH1, and CD133/CD44), which are associated with osimertinib resistance. We also demonstrated that beclin 1 help prevent the protein degradation of Sox 2 and ALDH to maintain stemness. These findings support a new physiological role for Beclin 1-dependent alternative macroautophagy in stem-like cell maintenance. Therefore, we hypothesized that Beclin 1 is beneficial for the maintenance of cancer stem-like cells by preventing the protein degradation of Sox2 and ALDH1.

Several studies have reported the role of autophagy in control of stemness of cancer cells. Autophagy maintains the stemness of ovarian cancer stem cells through regulation of FOXA2 (Peng et al, 2017), and inhibition of autophagy reduces chemoresistance and tumorigenic potential of ovarian cancer stem cells (Pagotto et al, 2017). Autophagy promotes the formation of vasculogenic mimicry by glioma stem cells through induction of KDR/VEGFR-2 activation (Wu et al, 2017). In acute myeloid leukemia stem cells, autophagy confers resistance to BET inhibitor JQ1 (Jang et al, 2017). Overall, these reports together with findings of the current study indicate that autophagy has a key role in maintenance 
of stemness of cancer cells, which then contribute to therapeutic resistance.

Since autophagy is important for osimertinib resistance as shown above, it is reasonable to ask

which autophagy genes are involved. Canonical autophagy is mediated by evolutionarily conserved autophagy-related genes (Atg genes), among which Atg5 is considered an essential component(Kim et al, 2013). Recently, Atg5-independent autophagy was reported. Both canonical and Atg5-independent non-canonical autophagic pathways have the same upstream autophagy initiation mechanism, regulated by several autophagic proteins, including Unc-51-like kinase 1 (Ulk1) and Beclin 1. Although higher expression levels of p-Beclin1 (Ser93) and Atg5 were observed in all osimertinib-resistant cell lines, osimertinib resistance was indeed inhibited by beclin 1 knockdown but not Atg5 silencing. This study firstly showed that Beclin 1-dependent and Atg5-independent alternative macroautophagy mediated osimertinib resistance.

\section{Conclusion}

In summary, this study delineates a previously unknown function of autophagy in promoting stemness and osimertinib resistance. Such findings are critical for devising a potential therapeutic strategy to overcome osimertinib resistance. In the future, more clinical work are needed to study whether autophagy level was enhanced in osimertinib-resistant patients and to test the efficacy of autophagy inhibition in combination with osimertinib in EGFR-mutant patients. 


\section{Materials and Methods}

\section{$388 \quad$ Cell lines}

PC-9 cells and gefitinib-resistant PC-9GR cells were generously provided by Prof. J. Xu and Dr. M. Liu

(Guangzhou Medical University, China). H1975 cells were from the American Type Culture Collection

(ATCC). To establish osimertinib-resistant cell lines, the parental cells were treated with osimertinib at

the concentration of $\mathrm{IC}_{50}$ for 2 weeks, with higher drug levels for another 3 weeks. The latter dosage was

sufficient to kill all parental cells. When resistant clones were visible, the cells were diluted to a single

cell per well, and continuous culture was performed in presence of osimertinib. All cells were cultured in

RPMI-1640 (Hyclone) with Earle's salts, supplemented with 10\% FBS (Gibco), 2 mmol/L L-glutamine

(Gibco), 100U/ml penicillin (HyClone), and $100 \mu \mathrm{g} / \mathrm{mL}$ streptomycin (Hyclone) at $37^{\circ} \mathrm{C}$, with $5 \% \mathrm{CO}_{2}$ and $90 \%$ humidity.

\section{Reagents}

Osimertinib (TAGRISSO) was obtained from Astra Zeneca. Spautin-1 (S7888), 3-Methyladenine

(S2767) and rapamycin (S1039) were purchased from Selleck. Cycloheximide (C6628) was purchased

from Sigma-Aldrich, and cycloheximide (HY-12320) from MedChem Express. Anti-LC3II (\#12741S),

SQSTM1/p62 (\#8025S), Atg5 (\#9980S), Beclin 1 (\#3495S), phospho-(Ser93)-beclin1 (\#14717S), Sox2

(\#3579S), ALDH1A1 (\#36671S), GAPDH (\#2118S) antibodies were from Cell Signaling Technology.

\section{Cell growth assays}

The MTT cell proliferation assay was performed as previously described(Yao et al, 2010). Briefly,

$2 \times 10^{3}$ cells/well were seeded in 96-well plates and treated with osimertinib or dimethyl sulfoxide 
(DMSO) 24 hours later. Absorbance was measured 72 hours after treatment. All experiments were repeated for at least three times. Cell proliferation was also assessed by the Ki67 incorporation assay with a Ki67 labeling and detection kit (BM2889, Boster). Briefly, cells were treated with osimertinib for 48h, incubated for 6h with Ki67 (1:200 dilution), and fixed. Cells were counterstained with 4', 6-diamidino-2-phenylindole (DAPI) and observed under a fluorescence microscope.

\section{Colony-formation assay}

Briefly, 500 cells were resuspended in culture medium and seeded in six-well plates. After 14 days of culture, the cells were fixed with $4 \%$ paraformaldehyde and stained with $0.1 \%$ crystal violet. Colonies with a diameter greater than $1 \mathrm{~mm}$ were counted. Triplicate samples were used in the experiment.

\section{Transmission electron microscopy (TEM)}

The cells were pre-fixed with $2.5 \%$ glutaraldehyde in $0.1 \mathrm{M}$ PBS $(\mathrm{pH} 7.4$ ) for $2 \mathrm{~h}$ at room temperature, and post-fixed with $1 \%$ osmium tetroxide for $2 \mathrm{~h}$. The samples were then dehydrated in increasing concentrations of ethanol (50\%, 70\% and 100\%) and acetone, and finally embedded in Araldite. Fifty to sixty nanometer sections were cut on a LKB-I ultramicrotome and transferred to copper grids, post-stained with uranyl acetate and lead citrate, and examined by Gatan JEM-1400 plus transmission electron microscopy.

\section{Whole-exome sequencing}

Whole-exome sequencing libraries were prepared with $3 \mathrm{mg}$ DNA. Exomes were captured using the NimbleGen SeqCap Non-Standard Material 110823-HG19-BEx-L2R-D03-EZ for whole exome 
sequencing, and libraries were hybridized to custom-designed biotinylated oligonucleotide probes

432 (Roche NimbleGen, USA) covering the target region sequence for target-capture sequencing. DNA

paired-end reads for WES and target-capture sequencing, respectively. Raw sequencing reads were

filtered to obtain clean reads, which were then aligned to human genome assembly HG19 with

Burrows-Wheeler Aligner (BWA) (Newman et al, 2016). Reads with multiple mapping loci in the

genome, and those with more than three mismatches, more than one gap, or a gap of more than 20 base

long were removed. Reads harboring an Indel within $5 \mathrm{bp}$ of the fragment ends were removed.

Duplicated reads derived from PCR amplification were marked with Picard tools

(http://broadinstitute.github.io/picard/). Local realignments and base-quality recalibrations were performed with the GATK software (https://www.broadinstitute.org/gatk/).

\section{Measurement of autophagic activity and autophagy flux}

Autophagic activity was monitored with the Cell-ID Green Autophagy Detection Kit (Enzo Life

Sciences, France). The Cell-ID Green autophagy dye serves as a selective marker of autolysosomes and

early autophagic compartments. Cells were trypsinized, washed with Assay Buffer, and incubated with

Cell-ID Green Detection Reagent for $30 \mathrm{~min}$ at room temperature, according to the manufacturer's instructions. Afterwards, 10,000 events/sample were analyzed by fluorescence microscopy. The 24-well plates were imaged on an ImageXpress Micro (Molecular Devices) high-throughput imager. Image analysis was performed with the MetaXpress software.

To measure autophagy flux, pBABE-EGFP-mCherry-MAP1LC3B (22418, deposited by Jayanta 
fresh media. We used ImageXpress Micro XLS Widefield High-Content screening System to automatic

scanning the fluorescent dots of autophagosomes labeled mCherry-EGFP-LC3 after an interval 12h.,

and the final time point was $72 \mathrm{~h}$. Autophagic flux was determinedby the yellow punta (the combination

457 of red and green fluorescence), and red punta (the extinction of EGFP in the acid environment of 458 lysosomes).

\section{Western blot}

461 Cells harvested by scraping were washed twice with PBS and lysed for $30 \mathrm{~min}$ at $4^{\circ} \mathrm{C}$ in RIPA buffer 462 (Sigma-Aldrich, France). After centrifugation at $12000 \times \mathrm{g}$ for $15 \mathrm{~min}$ at $4{ }^{\circ} \mathrm{C}$, the protein content was determined by the BCA assay. Equal amounts of protein were submitted to gel electrophoresis for $2 \mathrm{~h}$ at

$110 \mathrm{~V}$, followed by transfer onto PVDF membranes (90 min, $200 \mathrm{~mA}$ ) (Roche, Switzerland).

Membranes were blocked with 5\% bovine serum albumin (BSA) for $1 \mathrm{~h}$ at room temperature and incubated overnight at $4{ }^{\circ} \mathrm{C}$ with primary antibodies. Subsequently, the membranes were washed and incubated with $0.02 \mu \mathrm{g} / \mathrm{ml}$ horseradish peroxidase (HRP)-conjugated goat anti-rabbit or anti-mouse IgG

(Cell Signaling Technology, USA) for 1h, and visualized with ChemiDoc Touch System (Bio-Rad, USA).

\section{Pulmosphere formation assay}

472 For tumor pulmosphere formation, $1 \times 10^{3}$ tumor cells were seeded in non-adhesive 6-well plates. One 473 week after seeding, cell spheres characterized by tight, spherical, non-adherent colonies $>90 \mu \mathrm{m}$ in diameter were observed. All experiments were repeated at least three times. 
$476 \quad$ Flow cytometry

477 For CD133 and CD44 staining, $10^{6}$ cells were incubated with $10 \mu 1$ of each anti-CD133-PE (AC133 478 clone; Miltenyi Biotech) and anti-CD44-FITC (REA690 clone; Miltenyi Biotech) antibodies diluted in $479100 \mu \mathrm{l}$ of staining solution for $15 \mathrm{~min}$ at $4^{\circ} \mathrm{C}$. Then, $400 \mu \mathrm{l}$ buffer was added, and samples were analyzed 480 with the CytExpert software (Beckman Coulter, USA).

\section{siRNA Transfection}

483 Small interfering RNAs (siRNAs) were synthesized by Shanghai GenePharma Co., Ltd. (Shanghai, 484 China). For efficacy evaluation, $80 \mathrm{pmol}$ siRNA and negative control siRNA (siNC), respectively, were transfected into PC-9OR cells cultured in 6-well plates using Lipofectamine RNAiMAX, following the manufacturer's instructions. At $72 \mathrm{~h}$ post-transfection, knockdown efficiency was determined by examining endogenous expression by Western blot.

\section{Quantitative RT-PCR}

Total RNA was isolated from cultured cells with TRIzol reagent (\#15596026, Thermo Scientific, USA), and subjected to reverse transcription with PrimeScript RT reagent Kit containing gDNA Eraser (TAKARA, RR047A), according to the manufacturer's protocol. Quantitative RT-PCR was performed with fluorescent SYBR Green on a CFX96 Touch System (Bio-Rad, USA). Human GAPDH was used to normalize input cDNA.

\section{Xenografts}


Methods for xenograft implantation were described previously(Li et al, 2014). All animal protocols were approved by the Ethics Committee of the Third Military Medical University. Briefly, $2 \times 10^{6}$ PC-9GR cells were injected subcutaneously into the back, next to the left forelimb of 6-week-old female BALB/c A-nu mice (Laboratory Animal Center of Third Military Medical University, Chongqing, China), which all developed tumors of $\sim 30 \mathrm{~mm}^{3}$ within 5 to 7 days. The mice were then randomly assigned to 4 groups (8 mice/group), and treated with CQ (100 mg/L), osimertinib (20 mg/L), combined CQ and osimertinib, and drinking water (vehicle). The tumor volume was calculated as (length $\times$ width $\left.{ }^{2}\right) / 2$, and measured twice a week. The animals were maintained in individual ventilated cages in compliance with institutional guidelines. The animals were monitored for 8 weeks until euthanasia. For immunohistochemistry assay, tumor bearing mice in each group were sacrificed after 4 weeks of drug administration, and tumors were harvested, fixed with $4 \%$ paraformaldehyde, and paraffin embedded.

\section{Immunohistochemistry}

For immunohistochemistry, tumor sections were fixed with $4 \%$ paraformaldehyde overnight, paraffin embedded, sectioned, and stained with primary antibodies raised against Sox2 and LC3 (1:200). Sample scoring was performed by the H-score method that combines immunoreaction intensity and the percentage of tumor cells stained.

\section{Patient information, sample preparation and NGS sequencing}

A retrospective investigation was performed by profiling plasma or tissue biopsies from 39 patients with acquired resistance to osimertinib using NGS. This study was approved by the Institutional Review Board of Daping Hospital, Army military medical university. All patients were provided informed 
519 consent to this study and gave permission to the entire study. Disease progression was confirmed in each 520 patient according to Recist 1.1 criterion. Tissue DNA was extracted using QIAamp DNA FFPE tissue kit 521 (Qiagen) according to manufacturer's instructions. Circulating cell-free DNA was recovered from 4 to 5 $522 \mathrm{ml}$ of plasma using the QIAamp Circulating Nucleic Acid kit by Qiagen (Valencia, California, US).

523 DNA shearing was performed using Covaris M220. End repair and A tailing was followed by adaptor 524 ligation. The ligated fragments with size of 200-400 bp were selevted by beads (Agencourt AMPure XP 525 Kit, Beckman Coulter, California, US), hybridized with probe baits, selected by magnetic beads and 526 amplified by PCR. Indexed samples were sequenced on Nextseq500 sequencer (Illumina, Inc., USA) 527 with pair-end reads.

\section{Statistical analysis}

530 Statistical analysis was performed by GraphPad Prism 5 and the data were presented as mean \pm S.E.M. 531 The two-tailed Student' s t test was used to compare multiple sets of data. $\mathrm{P}<0.05$ was considered to be statistically remarkable. 


\section{Acknowledgments}

535 We thank Dr. Raffaella Sordella from Cold Spring Harbor Laboratory for kindly providing the valuable 536 cell lines used in the current study. We thank Prof. Xiang Xu and Prof. Xueqing Xu (both from Army 537 Medical University) for their kind advice about design of the study. This work was supported by the 538 Natural Science Foundation of China $(81672284,81472189,81672287,81702288$ and 81702291$)$ and a 539 foundation for PLA Young Scientists (16QNP106).

540

541 Competing interests: The authors disclosure no potential conflicts of interest. 


\section{References}

544 (2014) BRAF inhibitor resistance can be overcome by blocking autophagy. Cancer Discov 4: OF10

546 Akunuru S, James Zhai Q, Zheng Y (2012) Non-small cell lung cancer stem/progenitor cells are 547 enriched in multiple distinct phenotypic subpopulations and exhibit plasticity. Cell death \& disease 3: $548 \quad \mathrm{e} 352$

resistance in primary CLL lymphocytes. Leuk Res 35: 99-102

552

Auberger P, Puissant A (2017) Autophagy, a key mechanism of oncogenesis and resistance in leukemia.

Blood 129: 547-552

555

556

DNA profiling reveals heterogeneity of EGFR inhibitor resistance mechanisms in lung cancer patients.

Nature communications 7: 11815

Chen Y, Henson ES, Xiao W, Huang D, McMillan-Ward EM, Israels SJ, Gibson SB (2016a) Tyrosine

kinase receptor EGFR regulates the switch in cancer cells between cell survival and cell death induced

by autophagy in hypoxia. Autophagy 12: 1029-1046 
566 Chen Z, Teo AE, McCarty N (2016b) ROS-Induced CXCR4 Signaling Regulates Mantle Cell

Lymphoma (MCL) Cell Survival and Drug Resistance in the Bone Marrow Microenvironment via

Autophagy. Clinical cancer research : an official journal of the American Association for Cancer

Research 22: 187-199

570

Dogan I, Kawabata S, Bergbower E, Gills JJ, Ekmekci A, Wilson W, 3rd, Rudin CM, Dennis PA (2014)

SOX2 expression is an early event in a murine model of EGFR mutant lung cancer and promotes

proliferation of a subset of EGFR mutant lung adenocarcinoma cell lines. Lung cancer 85: 1-6

Eberlein CA, Stetson

D, Markovets AA, Al-Kadhimi KJ, Lai Z, Fisher PR, Meador CB, Spitzler P, Ichihara E, Ross SJ, Ahdesmaki MJ, Ahmed A, Ratcliffe LE, O'Brien EL, Barnes CH, Brown H, Smith

PD, Dry JR, Beran G, Thress KS, Dougherty B, Pao W, Cross DA (2015) Acquired Resistance to the 578

Mutant-Selective EGFR Inhibitor AZD9291 Is Associated with Increased Dependence on RAS 579

Signaling in Preclinical Models. Cancer research 75: 2489-2500

Gump JM, Staskiewicz L, Morgan MJ, Bamberg A, Riches DW, Thorburn A (2014) Autophagy variation

within a cell population determines cell fate through selective degradation of Fap-1. Nat Cell Biol 16:

Han J, Hou W, Goldstein LA, Lu C, Stolz DB, Yin XM, Rabinowich H (2008) Involvement of protective 
EGFR tyrosine kinase inhibitors activate autophagy as a cytoprotective response in human lung cancer

cells. PloS one 6: e18691

592

Hashida S, Yamamoto H, Shien K, Miyoshi Y, Ohtsuka T, Suzawa K, Watanabe M, Maki Y, Soh J, 594

Asano H, Tsukuda K, Miyoshi S, Toyooka S (2015) Acquisition of cancer stem cell-like properties in non-small cell lung cancer with acquired resistance to afatinib. Cancer science 106: 1377-1384

596

205-210

600

Nature communications 5: 4004

Honda S, Arakawa S, Nishida Y, Yamaguchi H, Ishii E, Shimizu S (2014) Ulk1-mediated Atg5-independent macroautophagy mediates elimination of mitochondria from embryonic reticulocytes.

Hu X, Shi S, Wang H, Yu X, Wang Q, Jiang S, Ju D, Ye L, Feng M (2017) Blocking autophagy improves the anti-tumor activity of afatinib in lung adenocarcinoma with activating EGFR mutations in vitro and in vivo. Scientific reports 7: 4559 
Jang JE, Eom JI, Jeung HK, Cheong JW, Lee JY, Kim JS, Min YH (2017) AMPK-ULK1-Mediated

Autophagy Confers Resistance to BET Inhibitor JQ1 in Acute Myeloid Leukemia Stem Cells. Clinical

cancer research : an official journal of the American Association for Cancer Research 23: 2781-2794

612

613

Janne PA, Yang JC, Kim DW, Planchard D, Ohe Y, Ramalingam SS, Ahn MJ, Kim SW, Su WC, Horn L,

Haggstrom D, Felip E, Kim JH, Frewer P, Cantarini M, Brown KH, Dickinson PA, Ghiorghiu S, Ranson

M (2015) AZD9291 in EGFR inhibitor-resistant non-small-cell lung cancer. The New England journal of

medicine 372: 1689-1699

617

618

Justilien V, Walsh MP, Ali SA, Thompson EA, Murray NR, Fields AP (2014) The PRKCI and SOX2 619 oncogenes are coamplified and cooperate to activate Hedgehog signaling in lung squamous cell carcinoma. Cancer Cell 25: 139-151

Jutten B, Keulers TG, Peeters HJM, Schaaf MBE, Savelkouls KGM, Compter I, Clarijs R, Schijns O,

Ackermans L, Teernstra OPM, Zonneveld MI, Colaris RME, Dubois L, Vooijs MA, Bussink J, Sotelo J,

Theys J, Lammering G, Rouschop KMA (2018) EGFRvIII expression triggers a metabolic dependency and therapeutic vulnerability sensitive to autophagy inhibition. Autophagy: 1-13

626

Kim IG, Kim SY, Choi SI, Lee JH, Kim KC, Cho EW (2014) Fibulin-3-mediated inhibition of epithelial-to-mesenchymal transition and self-renewal of ALDH+ lung cancer stem cells through IGF1R signaling. Oncogene 33: 3908-3917 
Kim J, Kim YC, Fang C, Russell RC, Kim JH, Fan W, Liu R, Zhong Q, Guan KL (2013) Differential regulation of distinct Vps34 complexes by AMPK in nutrient stress and autophagy. Cell 152: 290-303

Kim TM, Song A, Kim DW, Kim S, Ahn YO, Keam B, Jeon YK, Lee SH, Chung DH, Heo DS (2015)

Mechanisms of Acquired Resistance to AZD9291: A Mutation-Selective, Irreversible EGFR Inhibitor. Journal of thoracic oncology : official publication of the International Association for the Study of Lung Cancer 10: 1736-1744

Lee MH, Koh D, Na H, Ka NL, Kim S, Kim HJ, Hong S, Shin YK, Seong JK, Lee MO (2017) MTA1 is a novel regulator of autophagy that induces tamoxifen resistance in breast cancer cells. Autophagy: 0

Lee TG, Jeong EH, Kim SY, Kim HR, Kim CH (2015) The combination of irreversible EGFR TKIs and SAHA induces apoptosis and autophagy-mediated cell death to overcome acquired resistance in EGFR T790M-mutated lung cancer. International journal of cancer 136: 2717-2729

Li L, Han R, Xiao H, Lin C, Wang Y, Liu H, Li K, Chen H, Sun F, Yang Z, Jiang J, He Y (2014) Metformin sensitizes EGFR-TKI-resistant human lung cancer cells in vitro and in vivo through inhibition of IL-6 signaling and EMT reversal. Clinical cancer research : an official journal of the American Association for Cancer Research 20: 2714-2726

Liu YN, Chang TH, Tsai MF, Wu SG, Tsai TH, Chen HY, Yu SL, Yang JC, Shih JY (2015) IL-8 confers resistance to EGFR inhibitors by inducing stem cell properties in lung cancer. Oncotarget 6: 
Ma T, Li J, Xu Y, Yu C, Xu T, Wang H, Liu K, Cao N, Nie BM, Zhu SY, Xu S, Li K, Wei WG, Wu Y,

Guan KL, Ding S (2015) Atg5-independent autophagy regulates mitochondrial clearance and is essential

Mizuuchi H, Suda K, Murakami I, Sakai K, Sato K, Kobayashi Y, Shimoji M, Chiba M, Sesumi Y, 660

Tomizawa K, Takemoto T, Sekido Y, Nishio K, Mitsudomi T (2016) Oncogene swap as a novel mechanism of acquired resistance to epidermal growth factor receptor-tyrosine kinase inhibitor in lung cancer. Cancer science 107: 461-468

Mok TS, Wu YL, Ahn MJ, Garassino MC, Kim HR, Ramalingam SS, Shepherd FA, He Y, Akamatsu H,

Theelen WS, Lee CK, Sebastian M, Templeton

A, Mann H, Marotti M, Ghiorghiu S, 666

Papadimitrakopoulou VA, Investigators A (2017) Osimertinib or Platinum-Pemetrexed in EGFR 667 T790M-Positive Lung Cancer. The New England journal of medicine 376: 629-640

Newman AM, Lovejoy AF, Klass DM, Kurtz DM, Chabon JJ, Scherer F, Stehr H, Liu CL, Bratman SV,

Say C, Zhou L, Carter JN, West RB, Sledge GW, Shrager JB, Loo BW, Jr., Neal JW, Wakelee HA, Diehn

M, Alizadeh AA (2016) Integrated digital error suppression for improved detection of circulating tumor

DNA. Nature biotechnology 34: 547-555

Nguyen KS, Neal JW (2012) First-line treatment of EGFR-mutant non-small-cell lung cancer: the role 
of erlotinib and other tyrosine kinase inhibitors. Biologics : targets \& therapy 6: 337-345

677 Nishino M, Ozaki M, Hegab AE, Hamamoto J, Kagawa S, Arai D, Yasuda H, Naoki K, Soejima K, Saya 678 cell-like characteristics in human lung adenocarcinoma. Journal of Cancer 8: 1774-1785

680

Nukaga S, Yasuda H, Tsuchihara K, Hamamoto J, Masuzawa K, Kawada I, Naoki K, Matsumoto S, Mimaki S, Ikemura S, Goto K, Betsuyaku T, Soejima K (2017) Amplification of EGFR Wild-Type Alleles in Non-Small Cell Lung Cancer Cells Confers Acquired Resistance to Mutation-Selective EGFR Tyrosine Kinase Inhibitors. Cancer research 77: 2078-2089

Okudela K, Woo T, Mitsui H, Tajiri M, Masuda M, Ohashi K (2012) Expression of the potential cancer stem cell markers, CD133, CD44, ALDH1, and beta-catenin, in primary lung adenocarcinoma--their prognostic significance. Pathol Int 62: 792-801

Ortiz-Cuaran S, Scheffler M, Plenker D, Dahmen L, Scheel AH, Fernandez-Cuesta L, Meder L, Lovly CM, Persigehl T, Merkelbach-Bruse S, Bos M, Michels S, Fischer R, Albus K, Konig K, Schildhaus HU, Fassunke J, Ihle MA, Pasternack H, Heydt C, Becker C, Altmuller J, Ji H, Muller C, Florin A, Heuckmann JM, Nuernberg P, Ansen S, Heukamp LC, Berg J, Pao W, Peifer M, Buettner R, Wolf J, Thomas RK, Sos ML (2016) Heterogeneous Mechanisms of Primary and Acquired Resistance to Third-Generation EGFR Inhibitors. Clinical cancer research : an official journal of the American Association for Cancer Research 22: 4837-4847 
698 Pagotto A, Pilotto G, Mazzoldi EL, Nicoletto MO, Frezzini S, Pasto A, Amadori A (2017) Autophagy

inhibition reduces chemoresistance and tumorigenic potential of human ovarian cancer stem cells. Cell $700 \quad$ death \& disease 8: e2943

Pao W, Miller VA, Politi KA, Riely GJ, Somwar R, Zakowski MF, Kris MG, Varmus H (2005) Acquired

resistance of lung adenocarcinomas to gefitinib or erlotinib is associated with a second mutation in the

EGFR kinase domain. PLoS medicine 2: e73

Peng Q, Qin J, Zhang Y, Cheng X, Wang X, Lu W, Xie X, Zhang S (2017) Autophagy maintains the

stemness of ovarian cancer stem cells by FOXA2. Journal of experimental \& clinical cancer research :

CR 36: 171

709

710 Planchard D, Loriot Y, Andre F, Gobert A, Auger N, Lacroix L, Soria JC (2015) EGFR-independent mechanisms of acquired resistance to AZD9291 in EGFR T790M-positive NSCLC patients. Annals of oncology : official journal of the European Society for Medical Oncology 26: 2073-2078

Sarvi S, Mackinnon AC, Avlonitis N, Bradley M, Rintoul RC, Rassl DM, Wang W, Forbes SJ, Gregory

CD, Sethi T (2014) CD133+ cancer stem-like cells in small cell lung cancer are highly tumorigenic and 
719 Tsukuda K, Takigawa N, Kiura K, Gazdar AF, Lam WL, Miyoshi S (2013) Acquired resistance to EGFR 720 inhibitors is associated with a manifestation of stem cell-like properties in cancer cells. Cancer research

73: $3051-3061$

722

Skoulidis F, Papadimitrakopoulou VA (2017) Targeting the Gatekeeper: Osimertinib in EGFR T790M

Mutation-Positive Non-Small Cell Lung Cancer. Clinical cancer research : an official journal of the

American Association for Cancer Research 23: 618-622

726

727

Soria JC, Mok TS, Cappuzzo F, Janne PA (2012) EGFR-mutated oncogene-addicted non-small cell lung

cancer: current trends and future prospects. Cancer treatment reviews 38: 416-430

Soria JC, Ohe Y, Vansteenkiste J, Reungwetwattana T, Chewaskulyong B, Lee KH, Dechaphunkul A,

Imamura F, Nogami N, Kurata T, Okamoto I, Zhou C, Cho BC, Cheng Y, Cho EK, Voon PJ, Planchard

D, Su WC, Gray JE, Lee SM, Hodge R, Marotti M, Rukazenkov Y, Ramalingam SS, Investigators F

(2018) Osimertinib in Untreated EGFR-Mutated Advanced Non-Small-Cell Lung Cancer. The New

England journal of medicine 378: 113-125

736 Sterlacci W, Savic S, Fiegl M, Obermann E, Tzankov A (2014) Putative stem cell markers in non-small-cell lung cancer: a clinicopathologic characterization. Journal of thoracic oncology : official publication of the International Association for the Study of Lung Cancer 9: 41-49 
741 EGFR-independent autophagy induction with gefitinib and enhancement of its cytotoxic effect by 742 targeting autophagy with clarithromycin in non-small cell lung cancer cells. Biochem Biophys Res 743 Commun 461: 28-34

744

745 Tang ZH, Cao WX, Su MX, Chen X, Lu JJ (2017) Osimertinib induces autophagy and apoptosis via 746 reactive oxygen species generation in non-small cell lung cancer cells. Toxicol Appl Pharmacol 321: $747 \quad 18-26$

748

749

Thress KS, Paweletz CP, Felip E, Cho BC, Stetson D, Dougherty B, Lai Z, Markovets A, Vivancos A, 750 Kuang Y, Ercan D, Matthews SE, Cantarini M, Barrett JC, Janne PA, Oxnard GR (2015) Acquired 751 EGFR C797S mutation mediates resistance to AZD9291 in non-small cell lung cancer harboring EGFR 752 T790M. Nature medicine 21: 560-562

754 Wang Z, Du T, Dong X, Li Z, Wu G, Zhang R (2016) Autophagy inhibition facilitates erlotinib cytotoxicity in lung cancer cells through modulation of endoplasmic reticulum stress. International 756 journal of oncology 48: 2558-2566

RW, Grishin NV, Peyton M, Minna J, Bhagat G, Levine B (2013) EGFR-mediated Beclin 1 
Wu HB, Yang S, Weng HY, Chen Q, Zhao XL, Fu WJ, Niu Q, Ping YF, Wang JM, Zhang X, Yao XH,

Bian XW (2017) Autophagy-induced KDR/VEGFR-2 activation promotes the formation of vasculogenic

mimicry by glioma stem cells. Autophagy 13: $1528-1542$

766

767

Yao Z, Fenoglio S, Gao DC, Camiolo M, Stiles B, Lindsted T, Schlederer M, Johns C, Altorki N, Mittal

V, Kenner L, Sordella R (2010) TGF-beta IL-6 axis mediates selective and adaptive mechanisms of 769

resistance to molecular targeted therapy in lung cancer. Proc Natl Acad Sci U S A 107: 15535-15540

770

771

Ye M, Wang S, Wan T, Jiang R, Qiu Y, Pei L, Pang N, Huang Y, Huang Y, Zhang Z, Yang L (2017)

Combined Inhibitions of Glycolysis and AKT/autophagy Can Overcome Resistance to EGFR-targeted

Therapy of Lung Cancer. Journal of Cancer 8: 3774-3784

Yeo SK, Wen J, Chen S, Guan JL (2016) Autophagy Differentially Regulates Distinct Breast Cancer

Stem-like Cells in Murine Models via EGFR/Stat3 and Tgfbeta/Smad Signaling. Cancer research 76:

$3397-3410$

Yu HA, Arcila ME, Rekhtman N, Sima CS, Zakowski MF, Pao W, Kris MG, Miller VA, Ladanyi M,

Riely GJ (2013) Analysis of tumor specimens at the time of acquired resistance to EGFR-TKI therapy in

155 patients with EGFR-mutant lung cancers. Clinical cancer research : an official journal of the American Association for Cancer Research 19: 2240-2247

Yu L, Chen Y, Tooze SA (2017) Autophagy pathway: cellular and molecular mechanisms. Autophagy: 0 
786 Yuan J, Zhang N, Yin L, Zhu H, Zhang L, Zhou L, Yang M (2017) Clinical Implications of the 787 Autophagy Core Gene Variations in Advanced Lung Adenocarcinoma Treated with Gefitinib. Scientific $788 \quad$ reports 7: 17814

789

790 Zeng X, Zhao H, Li Y, Fan J, Sun Y, Wang S, Wang Z, Song P, Ju D (2015) Targeting Hedgehog 791 signaling pathway and autophagy overcomes drug resistance of BCR-ABL-positive chronic myeloid 792 leukemia. Autophagy 11: 355-372

793

794 Zou Y, Ling YH, Sironi J, Schwartz EL, Perez-Soler R, Piperdi B (2013) The autophagy inhibitor 795 chloroquine overcomes the innate resistance of wild-type EGFR non-small-cell lung cancer cells to 796 erlotinib. Journal of thoracic oncology : official publication of the International Association for the 797 Study of Lung Cancer 8: 693-702

798

799

800

801 
803 Figure 1. Autophagy inhibition resulted in increased osimertinib sensitivity in osimertinib-sensitive cells. (A) Fluorescent micrographs of autophagosomes in PC-9GR and PC-9 cells treated with or without osimertinib for $24 \mathrm{~h}$. Scale bar, $10 \mu \mathrm{m}$. (B) Western blot showing that high autophagy flux was found in PC-9 cells treated with osimertinib. (C) Osimertinib treatment induced autophagy to a much greater extent than that of gefitinib in both PC-9 cells and PC-9GR cells. Gefitinib, 10nM in PC-9 cells and $4 \mu \mathrm{M}$ in PC-9GR cells; osimertinib, 20nM in PC-9 cells and 10M in PC-9GR cells. The level of LC3 was examined using Western blot. (D) MTT assay for PC-9GR and PC-9 cells treated with the indicated concentrations of osimertinib for $72 \mathrm{~h}$. Experiments were performed in triplicate, and data are mean \pm SEM. (E) Ki67 staining of PC-9GR cells treated with osimertinib with or without spautin-1(10 $\mu \mathrm{M})$. Scale bar, $100 \mu \mathrm{m}$. Experiments were performed in triplicate, and data are mean \pm SEM. Histogram shows the percentages of Ki67-positive cells in the indicated groups $\left({ }^{*} \mathrm{p}<0.05\right.$ by Student's $t$ test) (F) MTT assay for PC-9GR cells treated with the indicated concentrations of osimertinib with or without rapamycin $(500 \mathrm{nM})$ for $48 \mathrm{~h}$. Experiments were performed in triplicate, and data are mean $\pm \mathrm{SEM}$ (*p<0.05 by Student's t test). (G) Western blot assessment of PC-9GR cells treated with rapamycin for 48h. increasing concentrations of osimertinib for 48 hours. Experiments were performed in triplicate, and data are mean \pm SEM. Histogram shows IC50 values in the indicated groups $(* * p<0.01$ by Student's $t$ 
whole-exome sequencing.

Figure 3. Enhanced autophagy in osimertinib-resistant cells determines osimertinib resistance. (A)

Fluorescent micrographs of autophagic vacuoles in parental PC-9GR, PC-9, H1975 cells and the

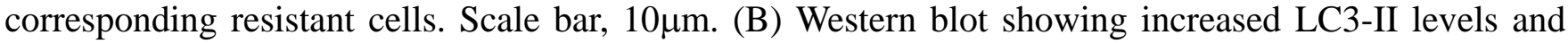
decreased p62 amounts in resistant cells. (C) MG132 treatment resulted in increased LC3-II levels in resistant PC-9OR3 cells when compared to that of PC-9 cells. (D) Micrographs obtained by transmission electron microscopy showing enhanced autophagosomes in resistant cells compared with parental

PC-9GR, PC-9, and H1975 cells. Magnification, $4 \times 10^{4}$. (E) The level of autophagy flux is increased in PC-9GROR cells. Representative images of mCherry-EGFP-LC3 vector were shown by fluorescent detection. The level of autophagy flux in PC-9GROR cells were increased compared with that in PC-9GR cells. Quantitative analysis of the number of yellow autophagosomesand red autolysosomes.

$* * \mathrm{P}<0.01$. (F) MTT assay for parental PC-9GR, PC-9, and H1975 cells and the corresponding resistant cells. Cells were treated with osimertinib with or without autophagy inhibitor, spautin-1 (10 $\mu \mathrm{M})$ for 72 values in the indicated groups $\left(* \mathrm{p}<0.05,{ }^{*} \mathrm{p}<0.01\right.$ by Student's $\mathrm{t}$ test $)$.

Figure 4. Osimertinib resistant cells show robust stem-cell like properties. (A) Parental and resistant 

and CD44-PE antibodies. Top left and top right quadrants represent CD133 positive populations, while top right and lower right are CD44 positive populations. The bar chart shows percentages in various groups $(\mathrm{n}=3, * \mathrm{p}<0.05$ by Student's t test). (C) Western blot showing the expression levels of the potential stem markers ALDH1 and Sox2 in osimertinib-resistant cells.

Figure 5. Loss of Sox 2 and ALDH1 affects stemness and osimertinib resistance. (A) MTT assay for PC-9OR3 cells transfected with control, Sox2, and ALDH1A1 siRNAs, respectively, treated with increasing concentrations of osimertinib for 48 hours. Experiments were performed in triplicate, and data are mean \pm SEM. Histogram shows IC50 values in the indicated groups $(* * \mathrm{p}<0.01$ by Student's $\mathrm{t}$ test). (B) Colony formation and pulmosphere formation assays for assessing the PC-9OR3 cell line after transfection with control, Sox2, and ALDH1A1 siRNAs, respectively. (C) CD133/CD44 positive cells were detected by flow cytometry with anti-CD133-FITC and CD44-PE antibodies. The bar chart shows percentages in various groups ( $\mathrm{n}=3, \mathrm{p}<0.05$ by Student's $\mathrm{t}$ test). (D) Western blot showing the expression levels of Sox2 and ALDH1 in the PC-9OR3 cell line after transfection with ALDH1A1 and Sox2 siRNAs, respectively.

Figure 6. Beclin 1-mediated, not Atg5-dependent autophagy is critical for stem-cell like properties and osimertinib resistance. (A) Pulmosphere formation assay for PC-9OR3 cells treated with DMSO or the autophagy inhibitor spautin-1 $(10 \mu \mathrm{M})$. (B) CD133/CD44 positive PC-9OR3 cells were detected after treatment with spautin-1 alone or combined with osimertinib. (C) ALDH1A1 and Sox2 levels measured by Western blot in PC-9OR3 cells after treatment with spautin-1 and osimertinib. (D) Phospho-beclin 1 
(Ser93), total beclin1, and Atg5 levels measured by Western blot in parental PC-9 and resistant PC-9OR3 cells. (E) Cell viability of PC-9OR3 cells transfected with control, beclin 1, and Atg5 siRNA, respectively. Experiments were performed in triplicate, and data are mean \pm SEM. (F) Colony formation and pulmosphere formation assays for PC-9OR3 cells after transfection with control, beclin 1, and Atg5 siRNAs, respectively. (G) CD133/CD44 positive PC-9OR3 cells were detected after transfection with control, beclin 1, and Atg5 siRNAs, respectively, by flow cytometry with anti-CD133-PITC and CD44-PE antibodies. (H) Sox2 and ALDH1 levels were measured in PC-9OR3 cells after transfection with control, beclin 1, and Atg5 siRNAs, respectively. (I) Sox2 and ALDH1 levels were measured in PC-9OR3 cells after transfection with control or beclin 1siRNAs following with MG132 treatment for 6h. (J) qPCR analysis of mRNA level of Sox2 and ALDH1 after beclin 1 knockdown.

Figure 7. Combination of the autophagy inhibitor CQ and osimertinib effectively inhibits the growth of PC-9GR xenografts. (A) PC-9GR xenografts were treated with control, CQ, osimertinib, and combined CQ/osimertinib, for 8 weeks. Tumor sizes were presented as mean \pm SEM $(n=8)$; n.s, not significant compared with the control group; $*, \mathrm{P}<0.001$ compared with the control group; $\dagger, \mathrm{P}<0.01$ compared with the control group; $\$, \mathrm{P}<0.05$ compared with the osimertinib alone group. (B) Body weight were presented as mean \pm SEM ( $\mathrm{n}=8$ ); n.s, not significant. (C) Representative immunohistochemical staining results for LC3 and Sox2, and hematoxylin-eosin staining for tumor xenografts from nude mice. (D) Whole protein cell lysates were prepared randomly from 3 tumors per group for Western blot to detect the indicated proteins.

Figure 8. Enhanced autophagy was found in patients with acquired resistance to osimertinib. (A) A chart 
890 showing potential resistance mechanisms to osimertinib in 39 NSCLC patients. More than 50\% patietns 891 developed resistance by yet unknown mechanisms. (B) Immunohistochemical staining results for LC3 892 and hematoxylin-eosin staining in paired tumor sections from 5 patients (before osimertinib treatment 893 and after osimertinib resistance). Positive staining was seen in patient \#1,4 and 5 after osiemrtinib 894 treatment. (C) Overall mutation spectrum of the 5 patients. Different color presents different types of 895 baseline mutation. The top bar demonstrated the number of mutations detected in an individual patient. 896 The side bar stands for the number of patients harboring the corresponding mutation. 
Figure 1
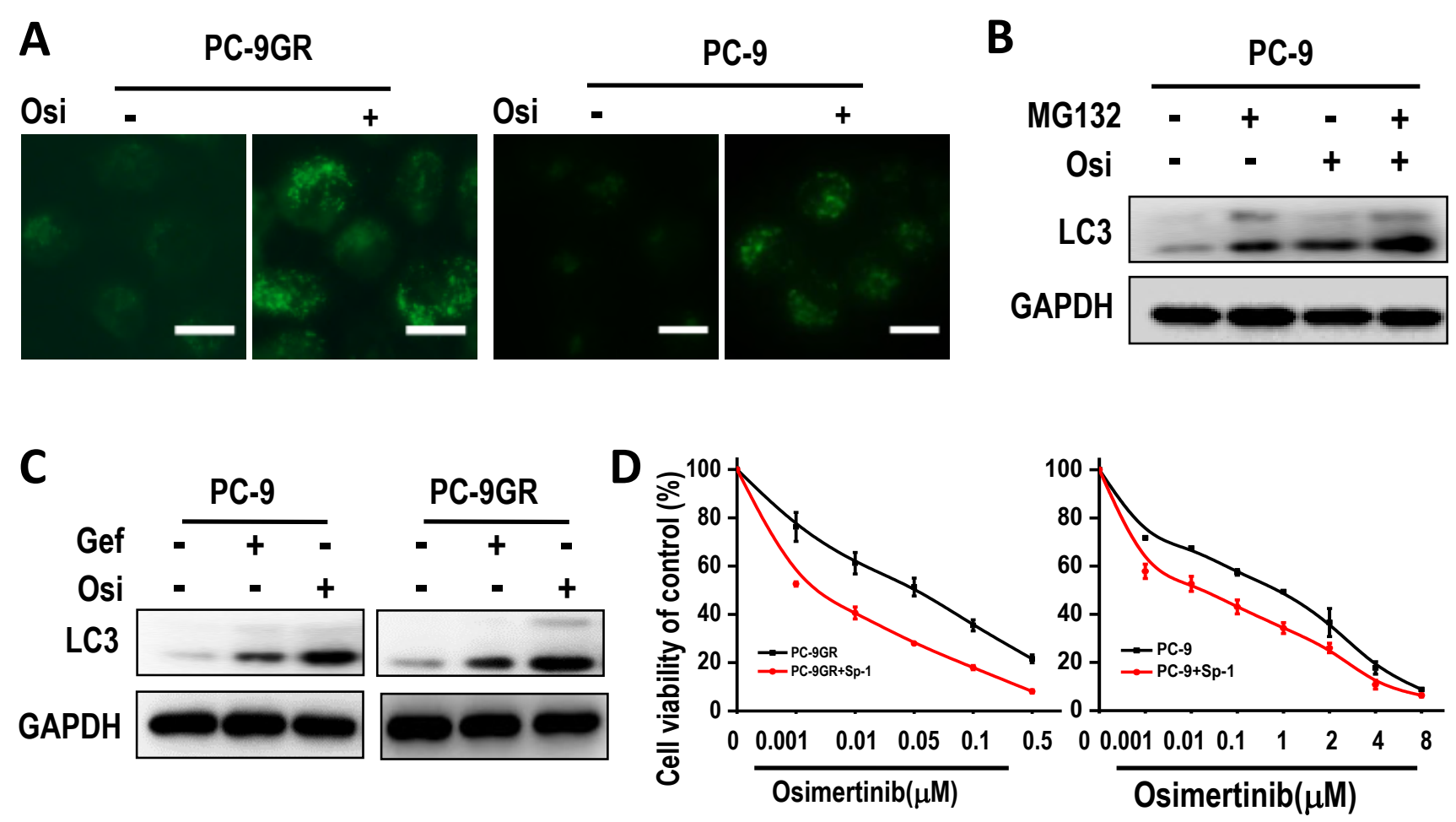
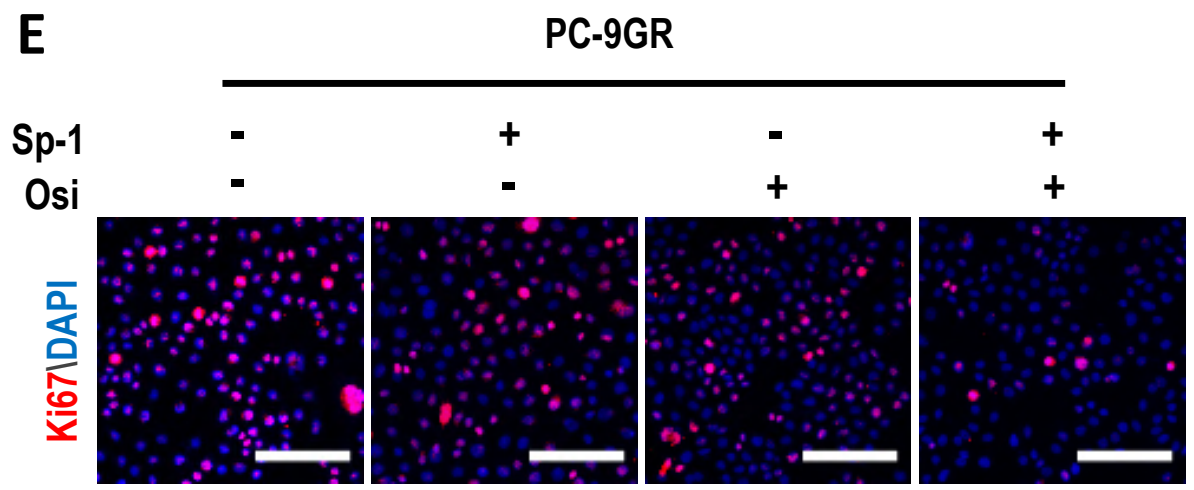

F

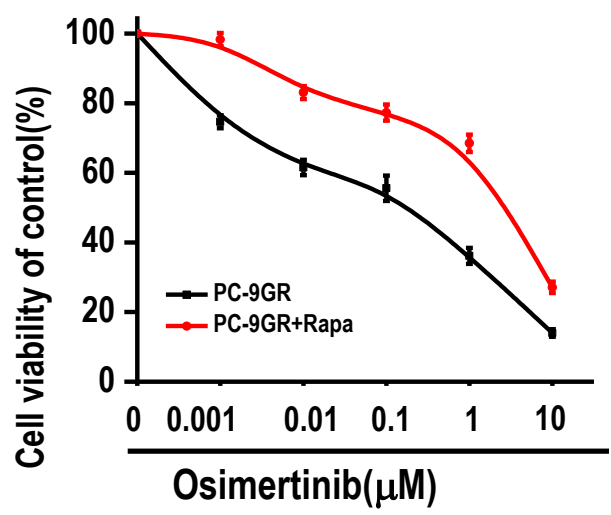

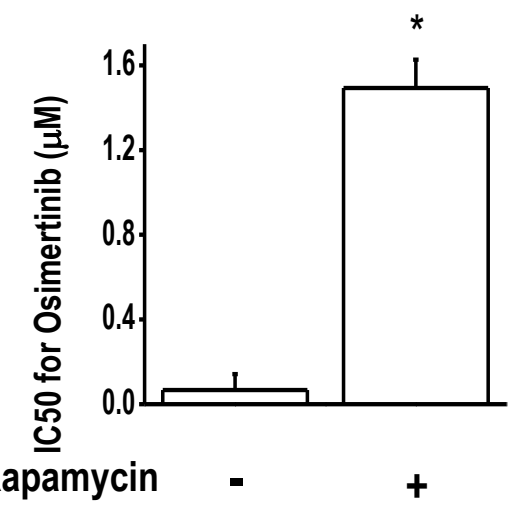

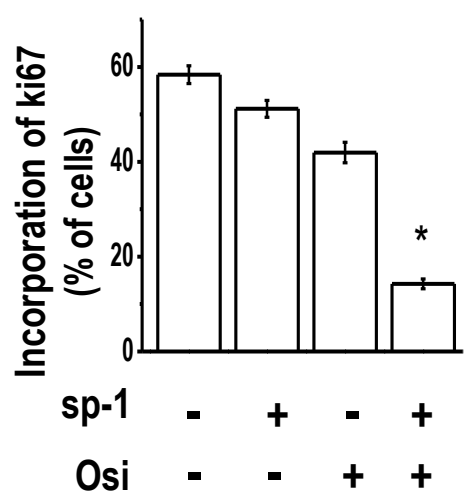

G

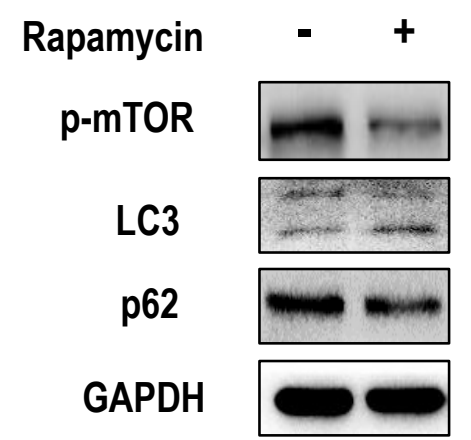




\section{Figure 2}

A

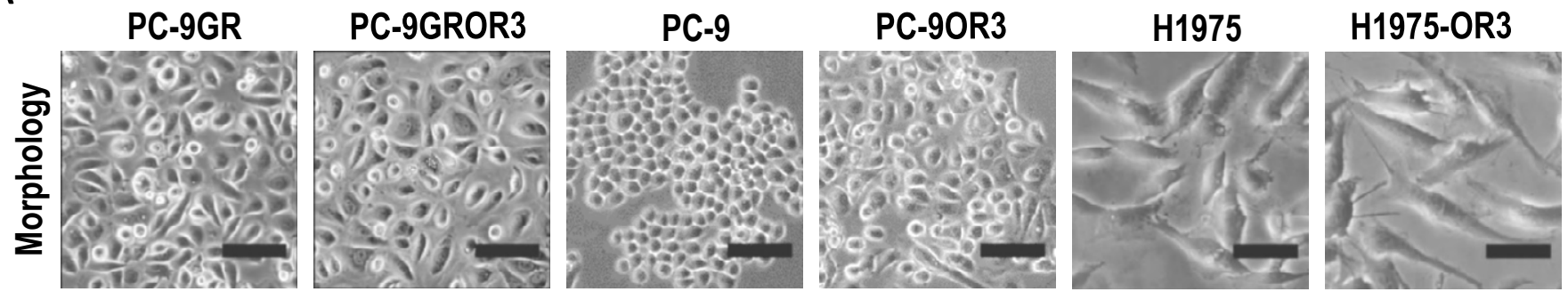

B

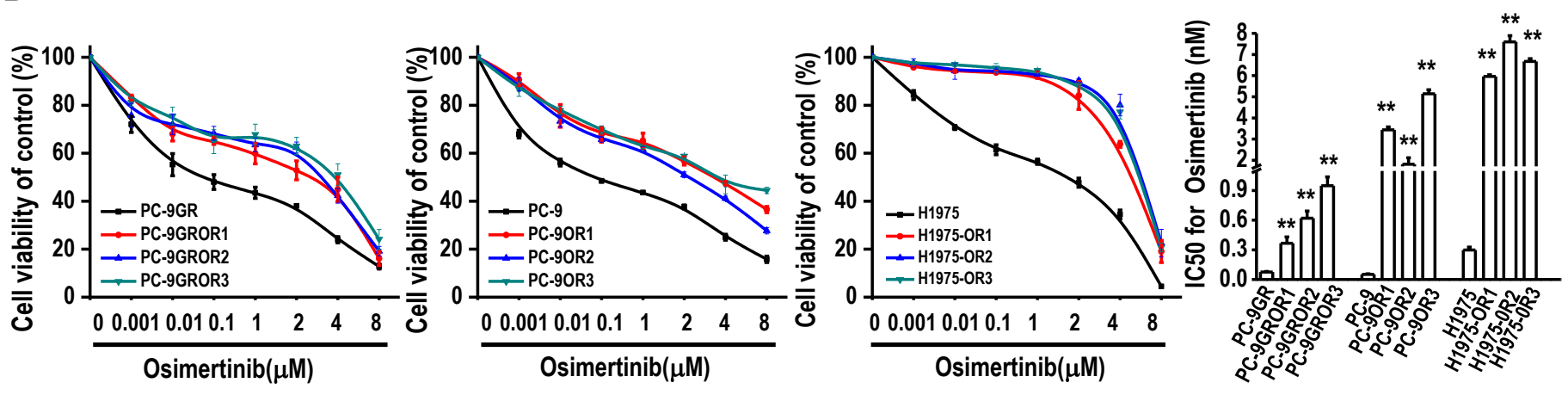

C

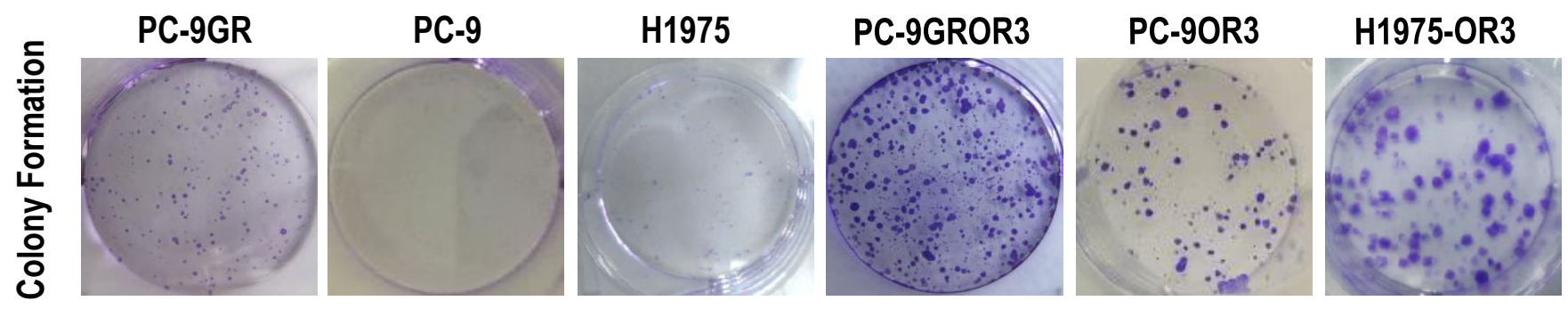

\begin{tabular}{|c|c|c|c|c|c|c|}
\hline cell lines & Del19 & L858R & T790M & EGFR amplification & Met amplification & BRAF amplification \\
\hline PC-9 & + & - & - & - & - & - \\
\hline PC-9OR1 & + & - & - & - & - & - \\
\hline PC-9OR2 & + & - & - & - & - & - \\
\hline PC-9OR3 & + & - & - & - & - & - \\
\hline PC-9GR & + & - & + & - & - & - \\
\hline PC-9GROR1 & + & - & + & - & - & - \\
\hline PC-9GROR2 & + & - & + & - & - & - \\
\hline PC-9GROR3 & + & - & - & + & - & - \\
\hline H1975 & - & + & + & - & - & - \\
\hline H1975-OR1 & - & + & + & - & + & + \\
\hline H1975-OR2 & - & + & + & - & + & - \\
\hline H1975-OR3 & - & + & + & - & + & - \\
\hline
\end{tabular}


D

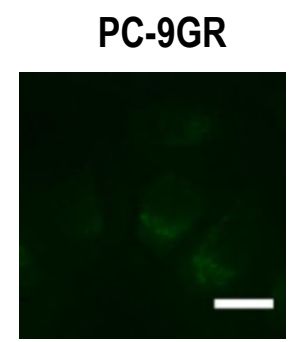

PC-9

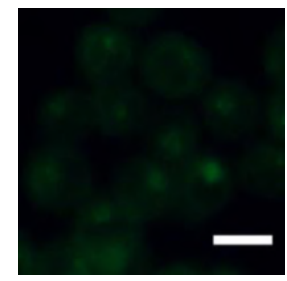

H1975
PC-9GROR3

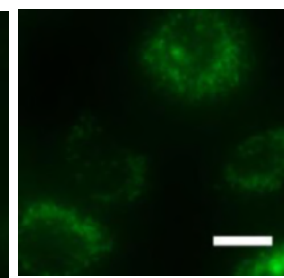

PC-9-OR3

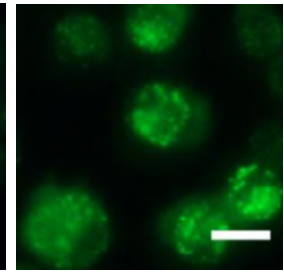

H1975-OR3
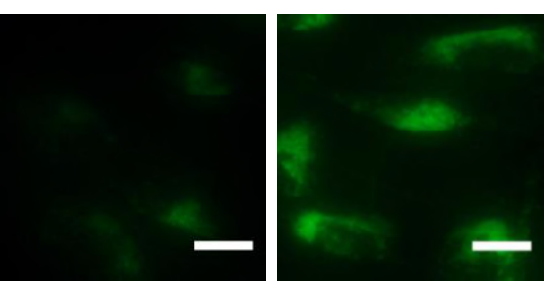

PC9-GR

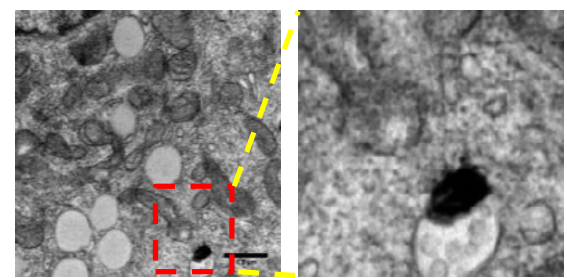

PC-9

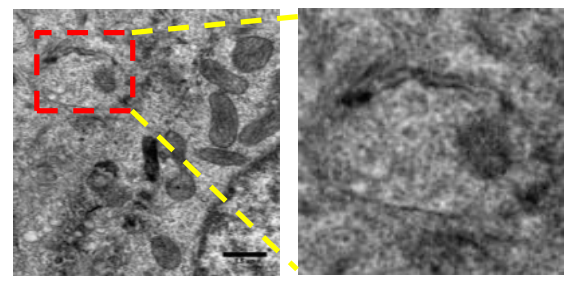

H1975
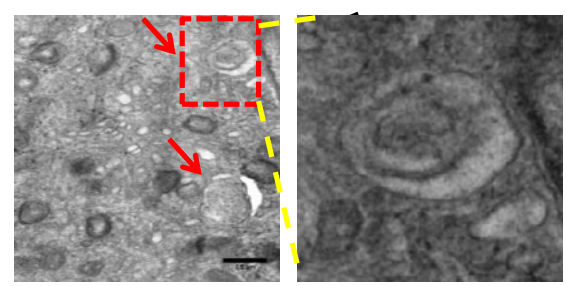

PC9-GROR3

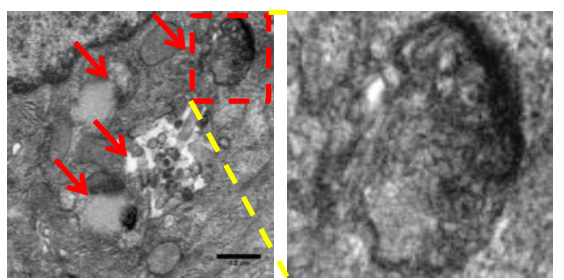

PC9-OR3

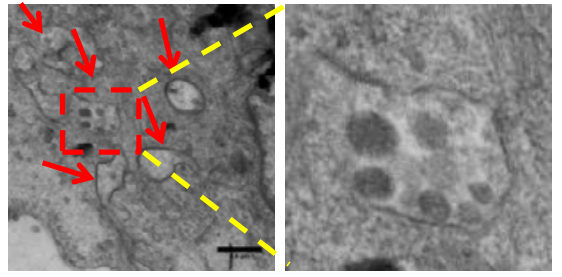

H1975-OR3

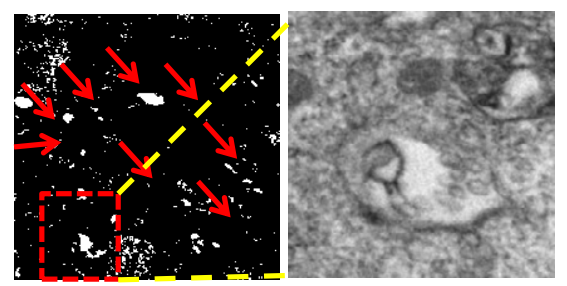

B

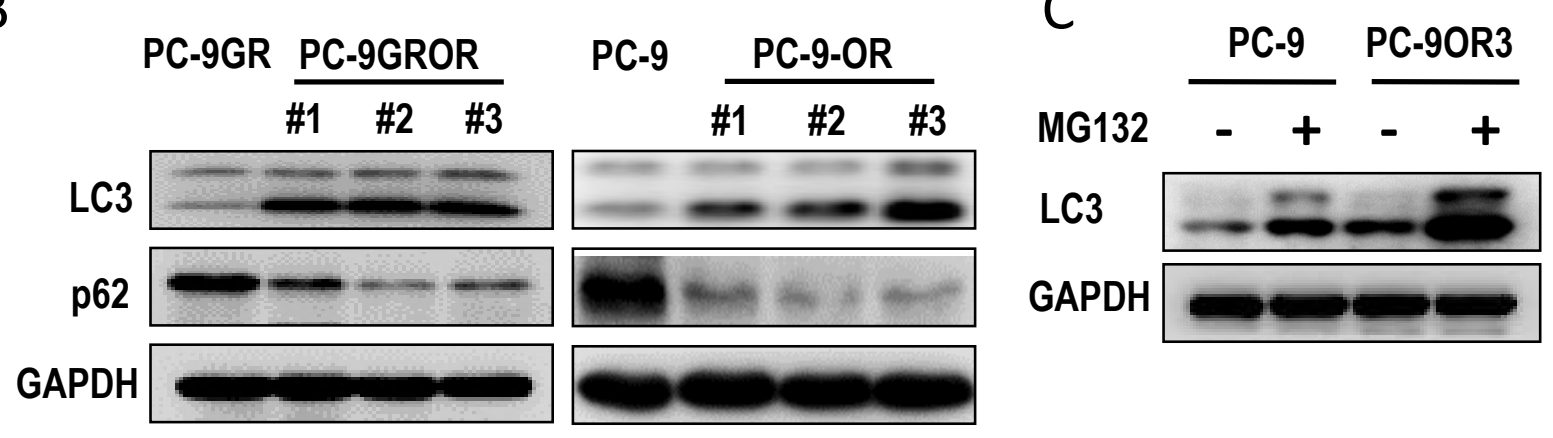

E
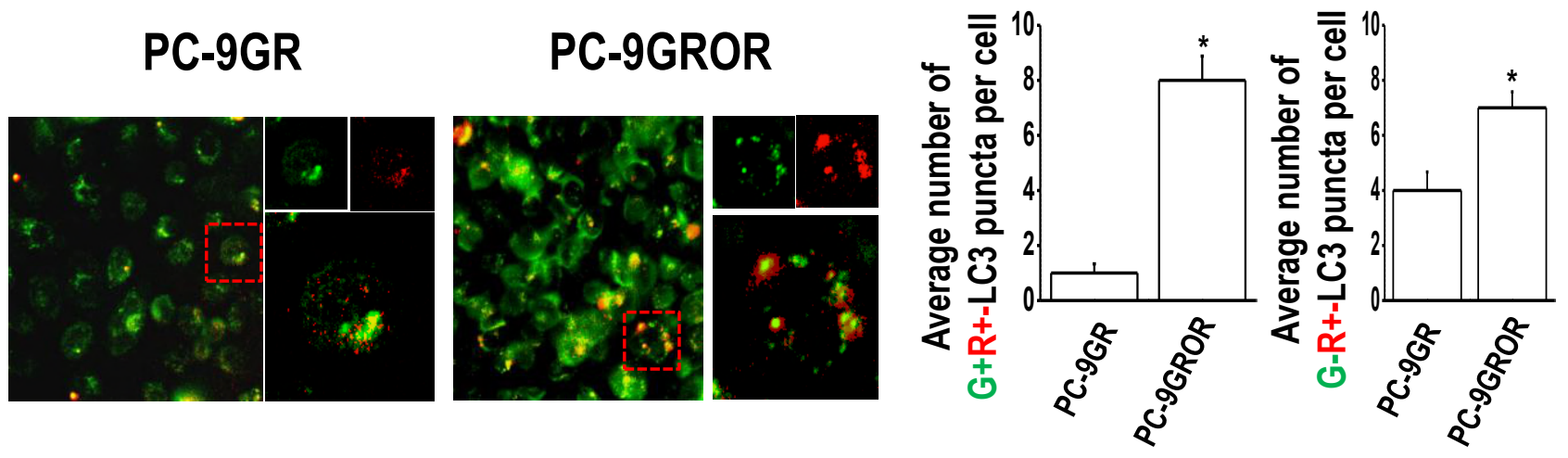

F
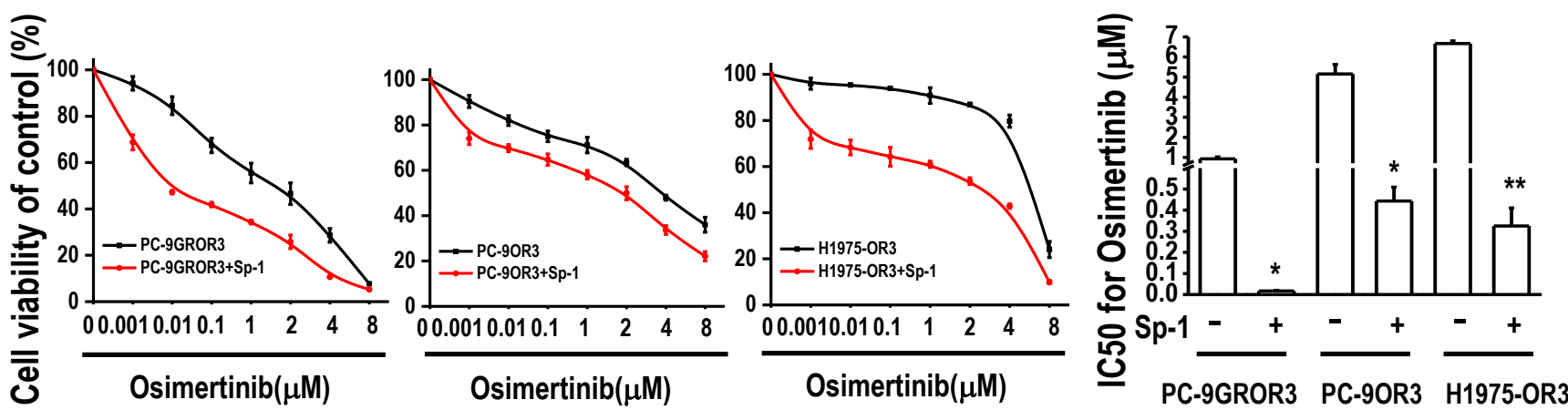
Figure 4
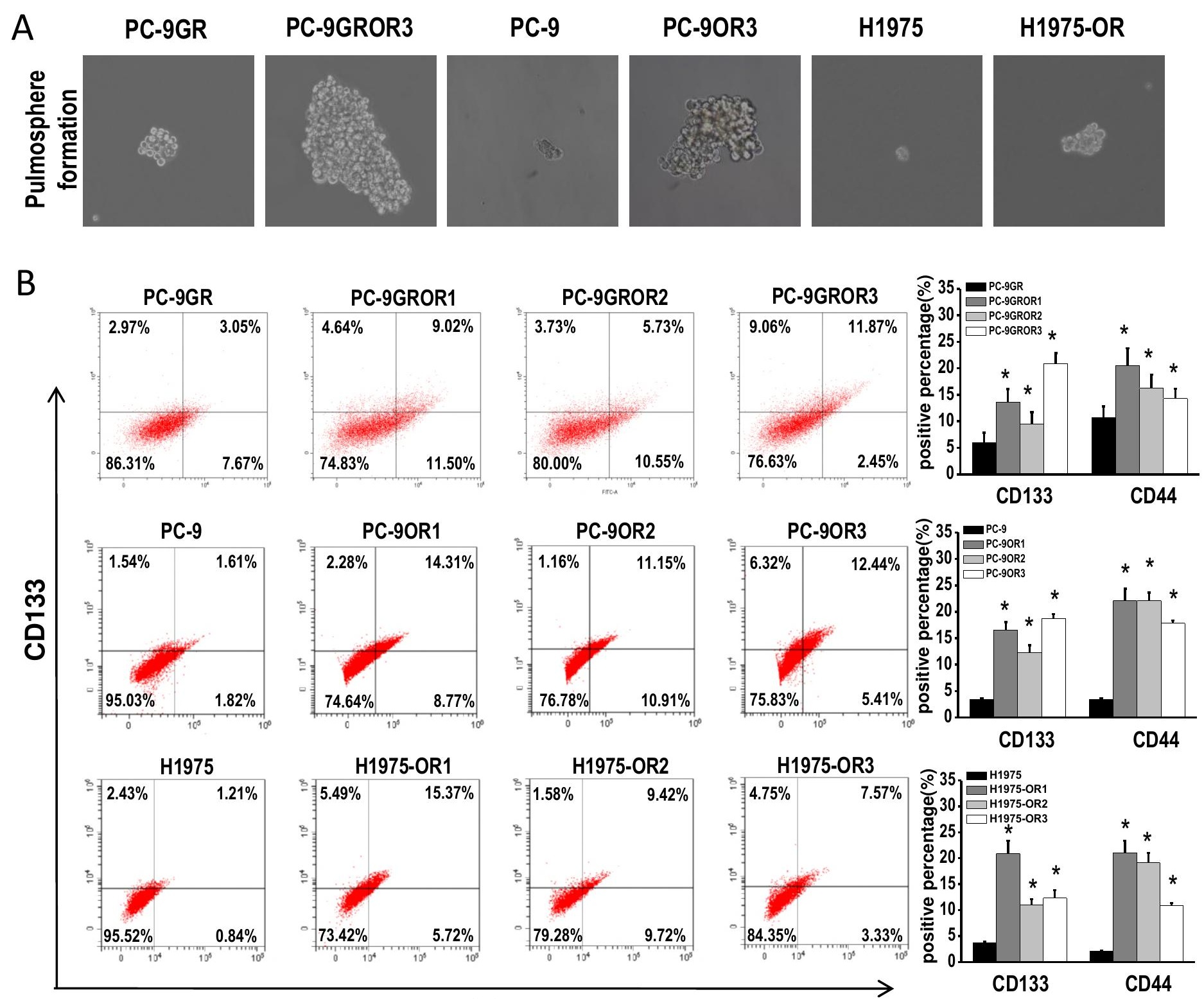

\section{CD44}

PC-9GR $\frac{\text { PC-9GROR }}{\# 1 \quad \# 2 \quad \# 3}$

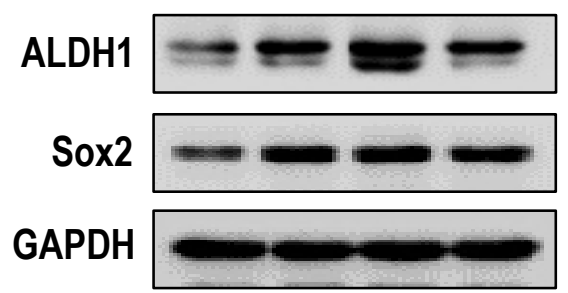

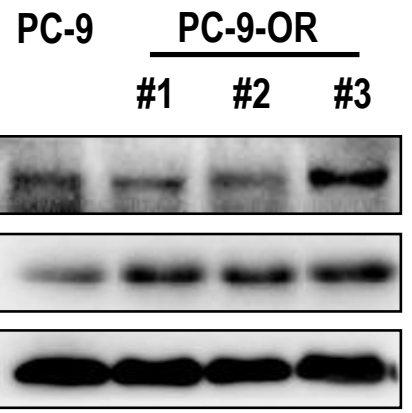




\section{Figure 5}

A

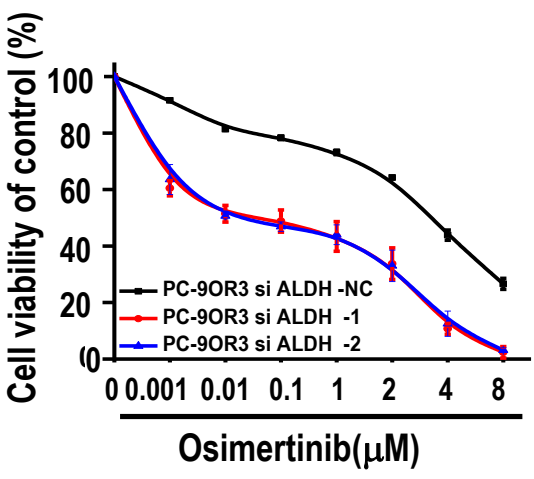

NC

\begin{tabular}{lr} 
& si Sox2 \\
\hline$\# 1$ & $\# 2$
\end{tabular}
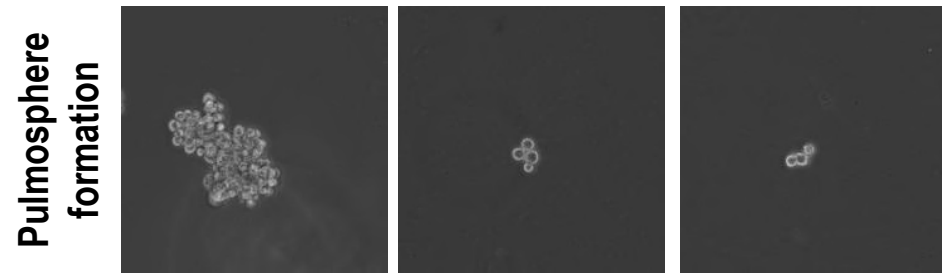

흥

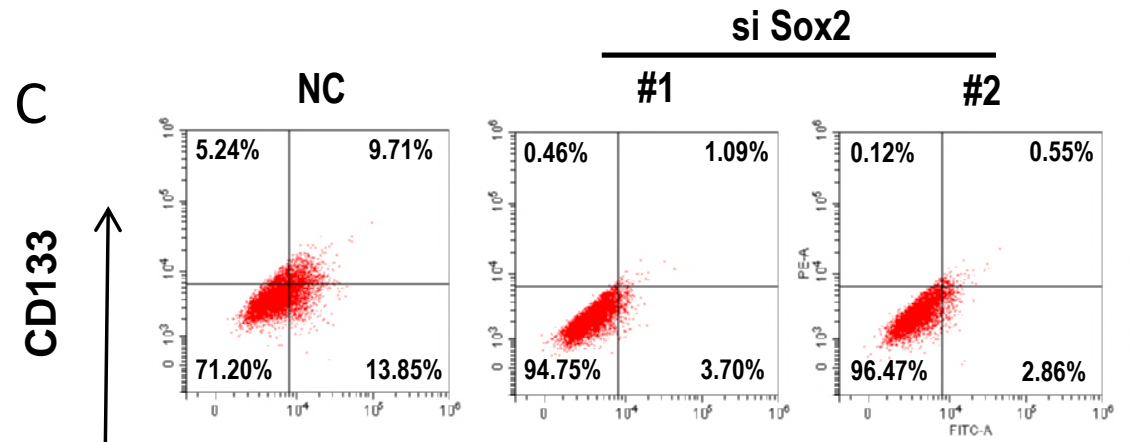

\section{CD44}

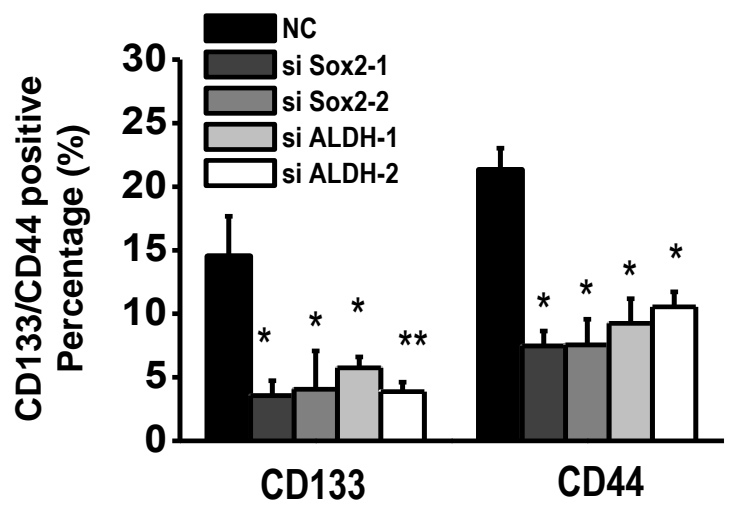

D
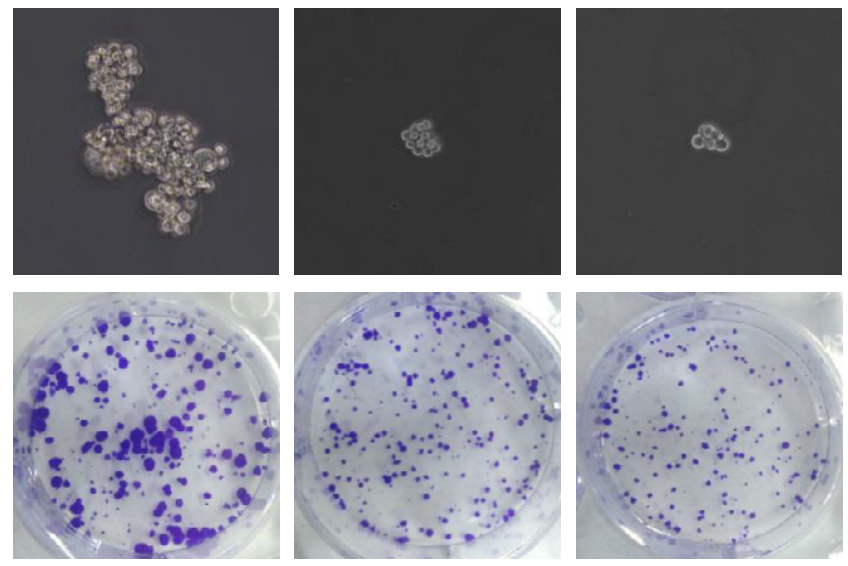

$\frac{\text { NC \#1 \#2 }}{\text { si Sox2 }} \frac{\text { NC \#1 \#2 }}{\text { si ALDH1 }}$

$\overline{\# 1 \quad \# 2}$

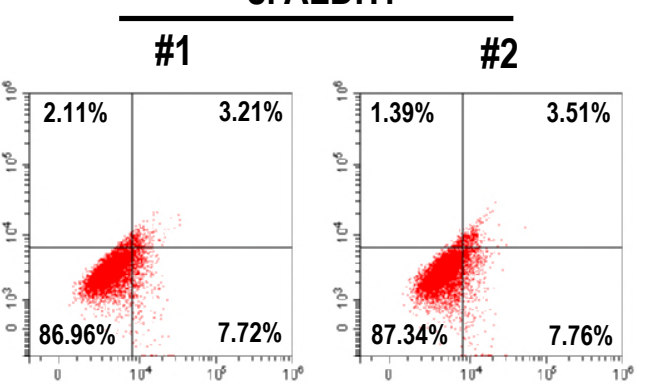


bioRxiv preprint doi: https://doi.org/10.1101/330092; this version pos 1 . The copyright holder for this preprint (which was not certified by peer review) is the author/funder. All rightspeserved. No reuse allowed without permission.

A

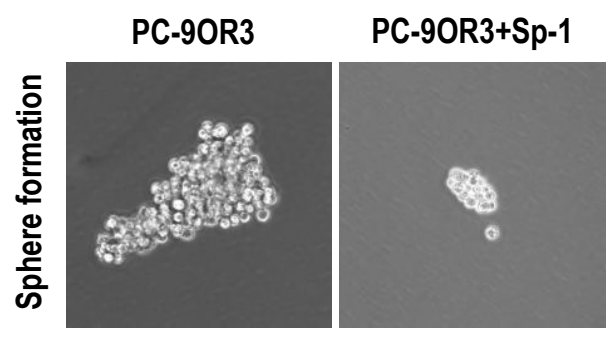

C

$\mathrm{F}$

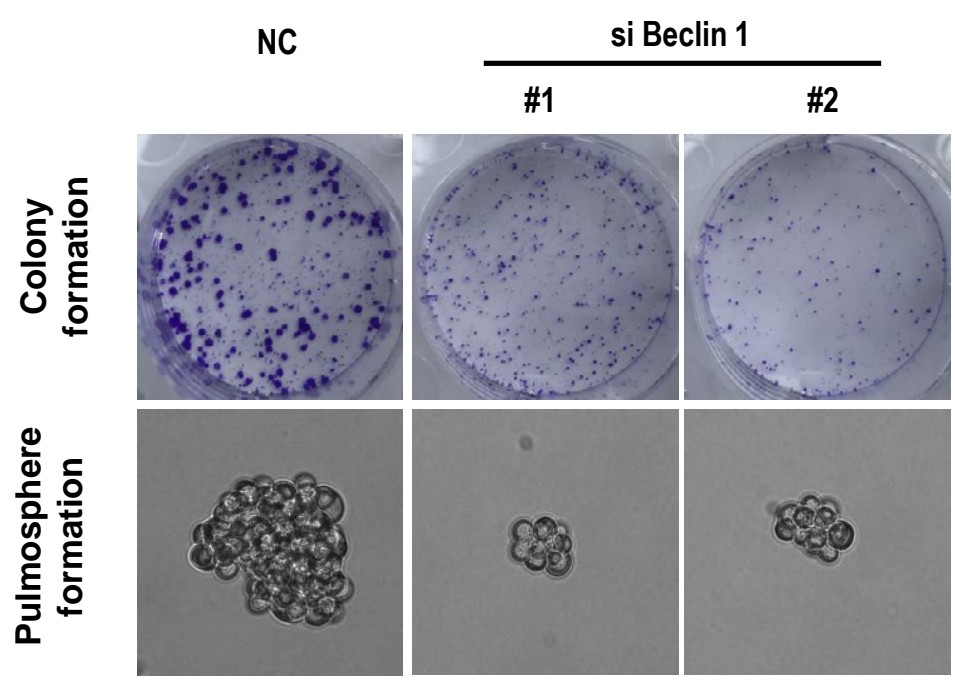

Beclin 1

Atg5

GAPDH
B

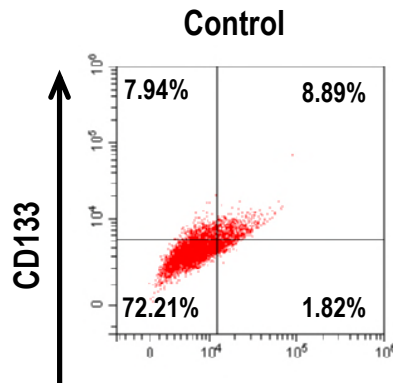

Osi

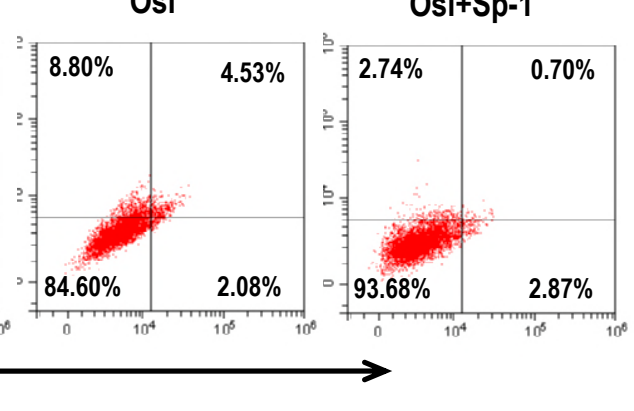

E

PC-9

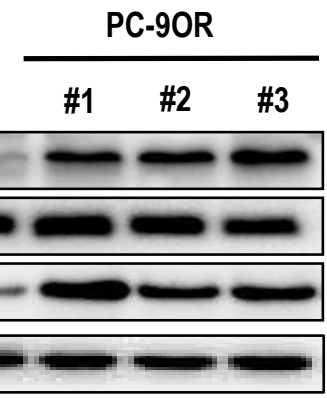

Sp-1

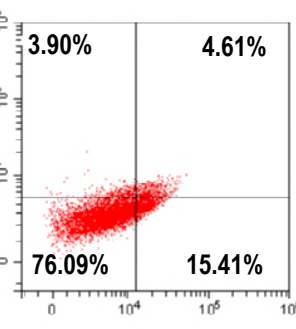

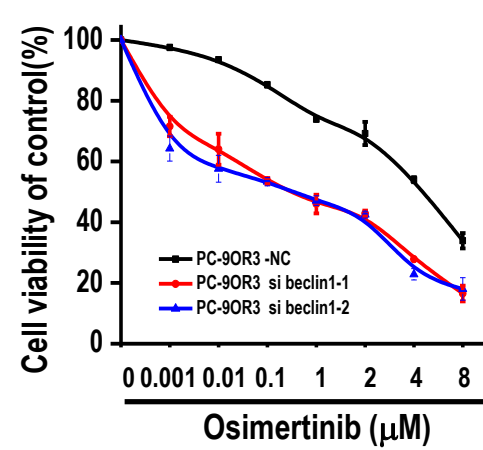

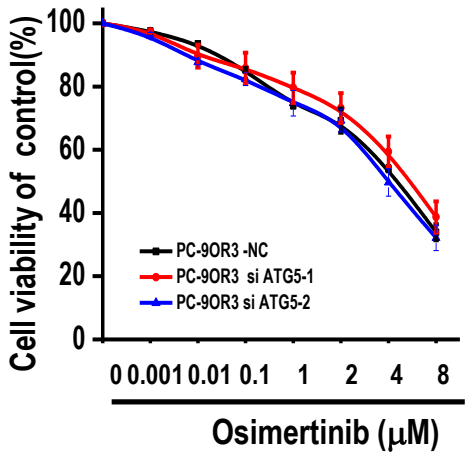

NC
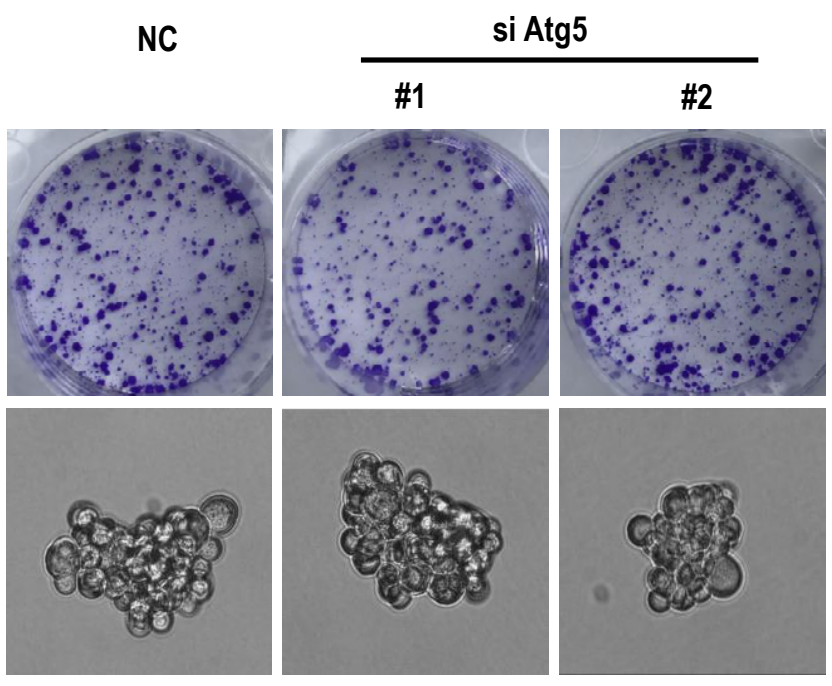

si Atg5

G

$\mathrm{H}$
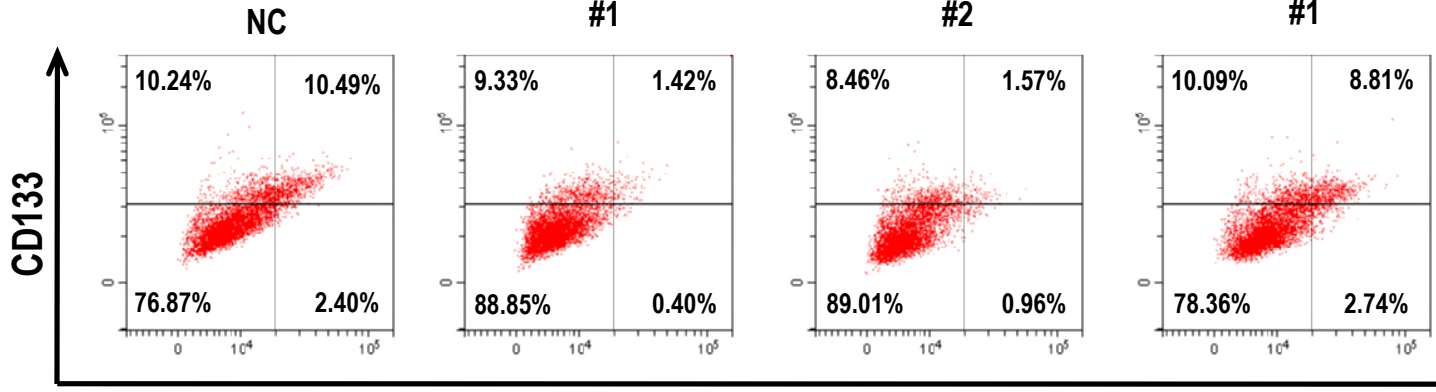

si Beclin 1

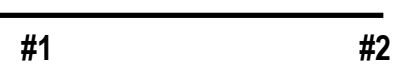

CD44
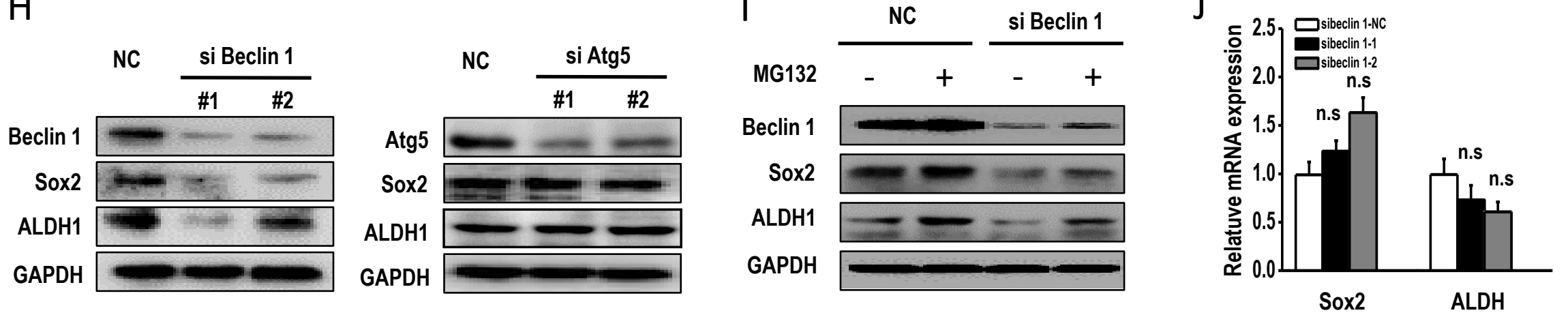


\section{Figure 7}

A

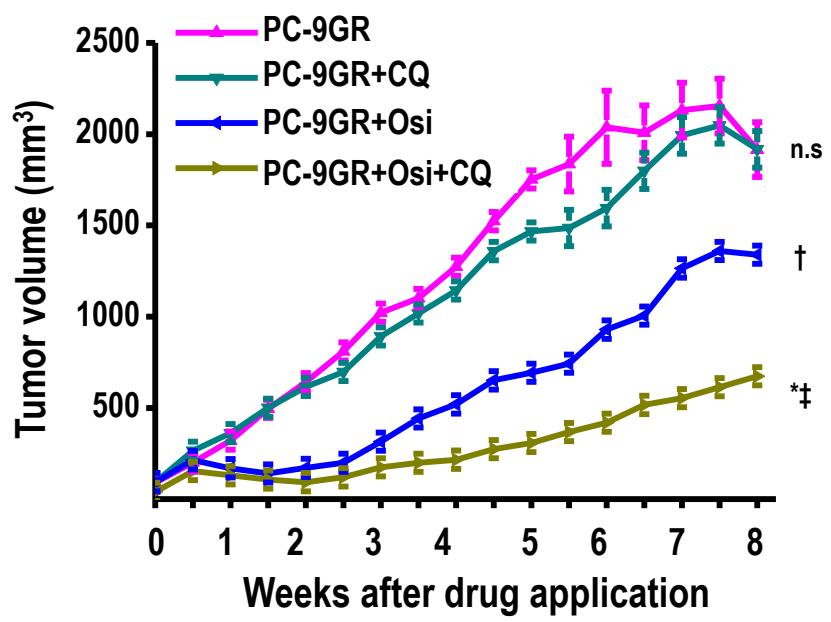

C

D
HE

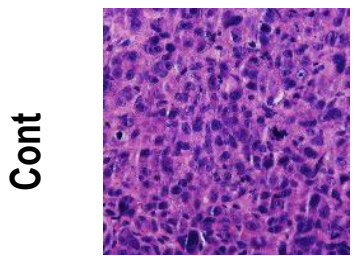

0

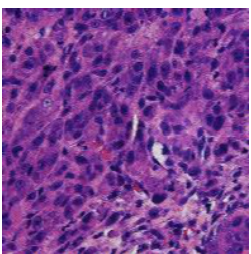

$\bar{s}$

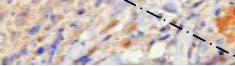

LC3
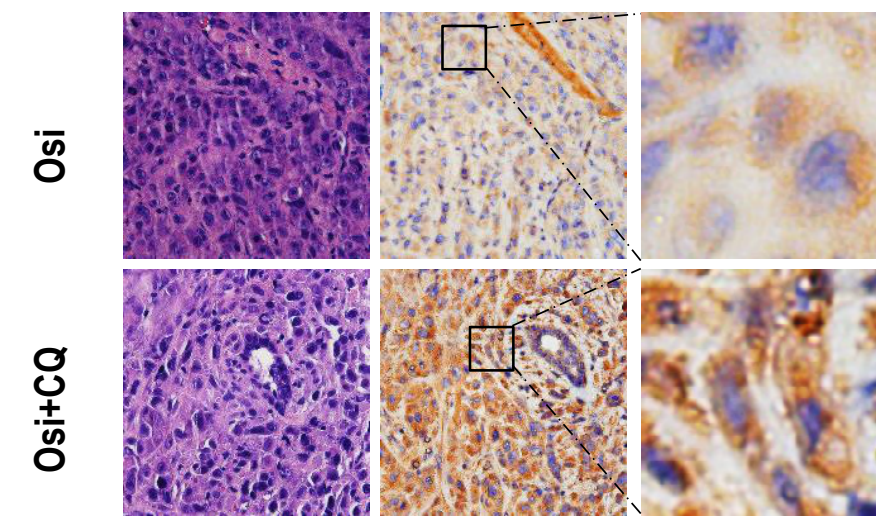

B

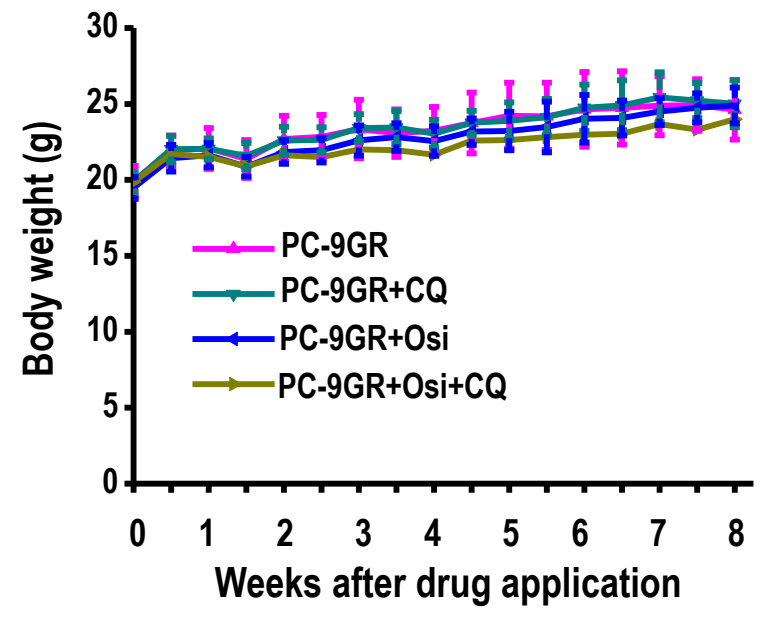

SOX2
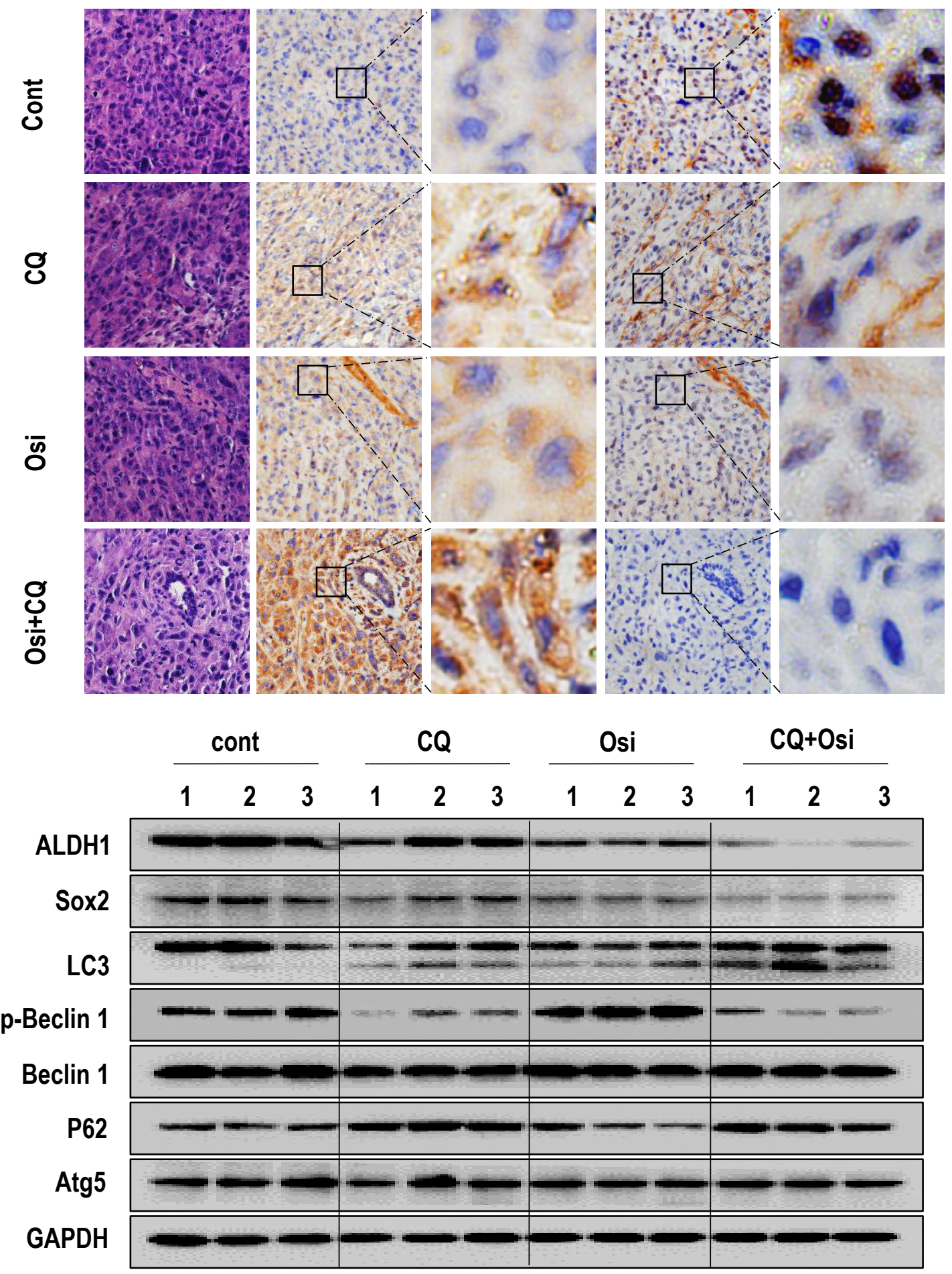


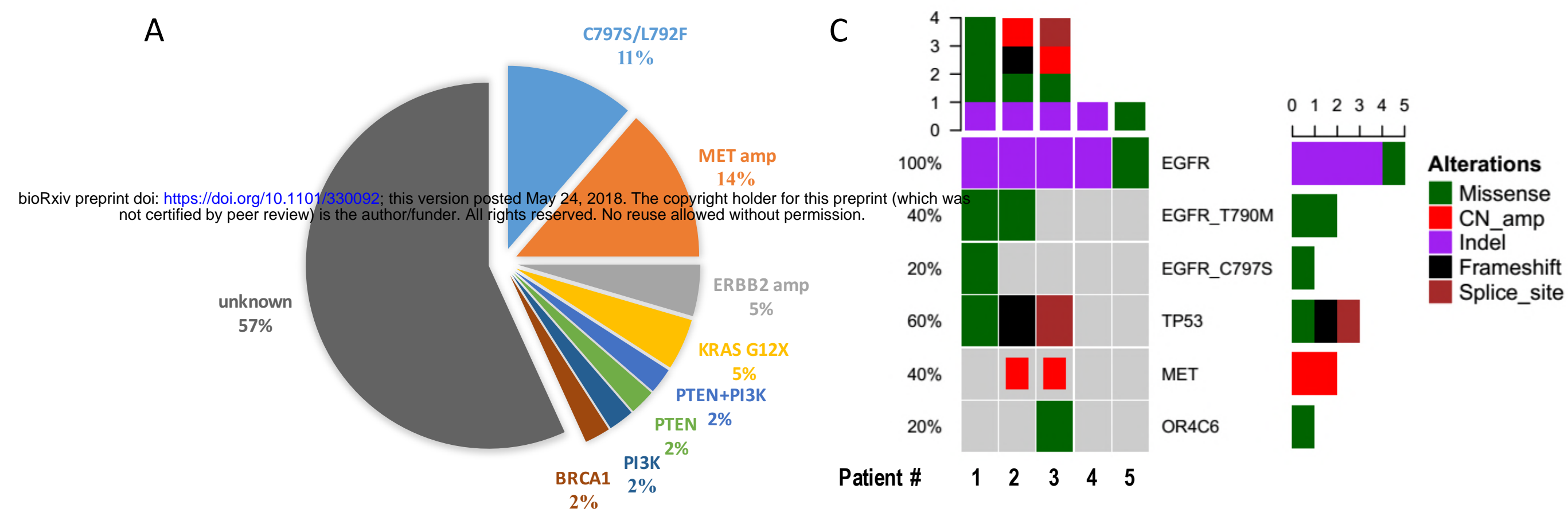

B

Before osimertinib usage

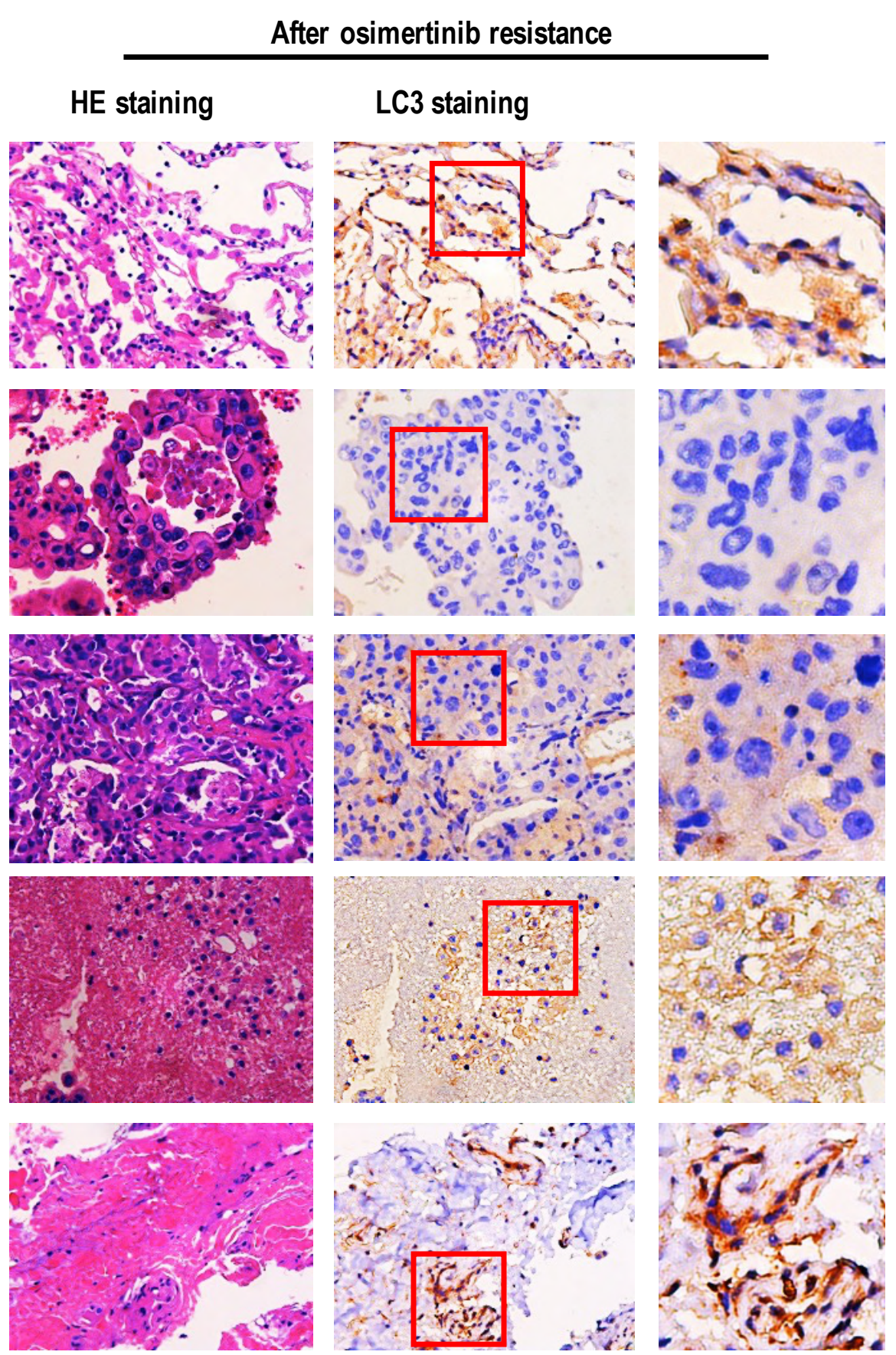

